\title{
The Chemical Composition of Praesepe (M44)
}

\author{
Ann Merchant Boesgaard ${ }^{1}$, Brian W. Roper ${ }^{1} \&$ Michael G. Lum ${ }^{1}$ \\ Institute for Astronomy, University of Hawai' $i$ at Manoa, \\ 2680 Woodlawn Drive, Honolulu, HI 96822 \\ boes@ifa.hawaii.edu \\ brianwroper@gmail.com \\ mikelum@ifa.hawaii.edu
}

\begin{abstract}
Star clusters have long been used to illuminate both stellar evolution and Galactic evolution. They also hold clues to the chemical and nucleosynthetic processes throughout the history of the Galaxy. We have taken high signal-tonoise, high-resolution spectra of 11 solar-type stars in the Praesepe open cluster to determine the chemical abundances of 16 elements: Li, C, O, Na, Mg, Al, Si, Ca, $\mathrm{Sc}, \mathrm{Ti}, \mathrm{V}, \mathrm{Cr}, \mathrm{Fe}, \mathrm{Ni}, \mathrm{Y}$, and Ba. We have determined Fe from Fe I and Fe II lines and find $[\mathrm{Fe} / \mathrm{H}]=+0.12 \pm 0.04$. We find that Li decreases with temperature due to increasing $\mathrm{Li}$ depletion in cooler stars; it matches the Li-temperature pattern found in the Hyades. The $[\mathrm{C} / \mathrm{Fe}]$ and $[\mathrm{O} / \mathrm{Fe}]$ abundances are below solar and lower than the field star samples due to the younger age of Praesepe (0.7 Gyr) than the field stars. The alpha-elements, $\mathrm{Mg}, \mathrm{Si}, \mathrm{Ca}$, and $\mathrm{Ti}$, have solar ratios with respect to Fe, and are also lower than the field star samples. The Fe-peak elements, $\mathrm{Cr}$ and $\mathrm{Ni}$, track Fe and have solar values. The neutron capture element $[\mathrm{Y} / \mathrm{Fe}]$ is found to be solar, but $[\mathrm{Ba} / \mathrm{Fe}]$ is enhanced relative to solar and to the field stars. Three Praesepe giants were studied by Carrera and Pancino; they are apparently enhanced in $\mathrm{Na}, \mathrm{Mg}$, and Ba relative to the Praesepe dwarfs. The $\mathrm{Na}$ enhancement may indicate proton-capture nucleosynthesis in the $\mathrm{Ne} \rightarrow \mathrm{Na}$ cycling with dredge-up into the atmospheres of the red giants.
\end{abstract}

Subject headings: stars: abundances; stars: evolution; stars: late-type; stars: solar-type; open clusters and associations: general; open clusters and associations: individual (M 44, NGC 2632, Praesepe); Galaxy: evolution

\footnotetext{
${ }^{1}$ Visiting Astronomer, W. M. Keck Observatory jointly operated by the California Institute of Technology and the University of California.
} 


\section{INTRODUCTION}

Open clusters have provided important information in the study of stellar evolution, Galactic chemical evolution, nucleosynthesis and light element abundances. The stars in a given cluster are formed from the pre-cluster gas with similar compositions and ages.

The Praesepe cluster is about the same age as the Hyades cluster at 0.7 Gyr (e.g. Salaris et al. 2004). Metallicity determinations cover a range of values. Boesgaard (1989) determined $[\mathrm{Fe} / \mathrm{H}]$ for several open clusters using sharp-lined cluster stars with the best high-resolution spectra for each cluster; for Praesepe she used the Palomar 5-m telescope and derived [Fe/H] $=+0.09 \pm 0.07$. Friel \& Boesgaard (1992) found $[\mathrm{Fe} / \mathrm{H}]=+0.04 \pm 0.04$ from six sharplined F dwarfs observed with CFHT high-resolution, high signal-to-noise spectra. With high-resolution spectra of four $\mathrm{G}$ dwarfs in Praesepe An et al. (2007) derived $[\mathrm{Fe} / \mathrm{H}]=$ $+0.11 \pm 0.03$. With spectra from VLT + UVES Pace et al. (2008) found abundances for 6 elements in 7 Praesepe dwarf stars; their Fe abundances are supersolar at $+0.27 \pm 0.10$. The compilation of Gratton (2000) gives $+0.04 \pm 0.06$ and Salaris et al. (2004) use $+0.13 \pm 0.06$ for Praesepe as one of their calibrating clusters. Carrera \& Pancino (2009) found $[\mathrm{Fe} / \mathrm{H}]=$ $+0.16 \pm 0.05$ for three red giant stars in Praesepe.

The Hyades and Praesepe clusters have long been thought to be so similar as to be coeval having formed in the same giant molecular cloud complex. In their study of the places of origin of 24 open clusters, Palous et al. (1977) find that for the angular rotational speeds of 13.5, 15.0, 17.5 and $20.0 \mathrm{~km} \mathrm{~s}^{-1} \mathrm{kpc}^{-1}$ Hyades and Praesepe were formed near each other. Their metallicities are similar at $[\mathrm{Fe} / \mathrm{H}] \sim+0.13$, their ages are similar at 0.7 Gyr (e.g. Salaris et al. 2004, Magrini et al. 2009) and their kinematic properties are similar (Eggen 1992).

There are some intriguing differences. The activity level in Praesepe is lower than that in the Hyades. ROSAT studies of the Hyades showed a detection rate of $90 \%$ for the G dwarfs in the Hyades (Stern et al. 1995), but only 33\% in the Praesepe G dwarfs (Randich \& Schmidt 1995). The dichotomy in the X-ray luminosity functions is not due to membership problems, sensitivity issues or differences in rotational velocity distributions (Barrado y Navascues et

al. 1998). Holland et al. (2000) and Franciosini et al. (2003) suggest that Praesepe may actually be two merging clusters of different ages. The former authors describe the main cluster with $630 \mathrm{M}_{\odot}$ extending to $12.1 \mathrm{pc}$ with a subcluster of $30 \mathrm{M}_{\odot}$ that is $3 \mathrm{pc}$ away from the center. There might be a possibility of somewhat different Fe abundances in the two merging pieces, but the major part is dominant by a factor of 21 in mass.

In this paper we present the abundances of 16 elments in 11 solar-temperature dwarfs in Praesepe from high-resolution, high signal-to-noise spectra obtained at the Keck I telescope 
with HIRES. We compare our results with those of Carrera \& Pancino (2011) who have determined chemical abundances in three red giants in Praesepe.

\section{OBSERVATIONS AND DATA REDUCTION}

The stars selected for this work are main sequence stars with colors and temperatures surrounding the solar value of $5774 \mathrm{~K}$. High-resolution spectra were obtained with HIRES (Vogt et al. 1994) on the Keck I telescope on Mauna Kea on two clear nights in January and February 2003. The spectral resolution is $\sim 45,000$ and the signal-to-noise ratios $(\mathrm{S} / \mathrm{N})$ range from 93 to 166 with a median of 153 . Typical integration times were $20-25$ minutes. The details of the observations of 11 Praesepe stars are given in Table 1. The spectral coverage is from $5730 \AA$ to $8140 \AA$ with some interorder gaps. On the night of 2003 January 11 (UT) we also took a $10 \mathrm{~s}$ exposure of the Moon to be used as a surrogate for the solar spectrum.

Each night we obtained 13 flatfield frames and 13 bias frames as well as Th-Ar spectra at the beginning and end of the night for wavelength calibration. The data reduction was done using standard IRAF1 routines. These include overscan-subtraction, bias-subtraction, master nightly flat-field normalizations, wavelength calibrations, scattered-light removal, cosmic ray removal, and continuum fitting. We were able to extract 19 orders of spectra.

In Figure 1 we show the color-magnitude diagram for Praesepe from UBV photometry done by Johnson (1952) and Mendoza (1967). The stars we observed in Table 1 are indicated by the open circles. All of our stars are confirmed members based on radial velocity measurements of Mermilliod \& Mayor (1999). All the stars are confirmed members (at 97-99\%) from proper motion measurements of Jones \& Cudworth (1983) and Jones \& Stauffer (1991), except KW 30 which they did not measure.

Figure 2 shows $60 \AA$ of spectrum for three of our stars which span a range in temperature. Several Fe I lines used in the analysis are indicated. The high quality of our data can be seen in this figure. It is easy to see that as the temperature decreases, the Fe I lines strength increases. Figure 3 shows the region near the Li I line for the same three stars. The Li I line decreases in strength going to cooler temperatures due to increasing depletion of Li. The three Fe I lines in that figure increase in strength with decreasing temperature. Figure 4 shows the region where the high excitation C I lines occur while Figure 5 is of the high excitation lines of the O I triplet in the same three stars.

\footnotetext{
${ }^{1}$ IRAF is distributed by the National Optical Astronomical Observatories, which are operated by AURA, Inc. under contract to the NSF.
} 


\section{ABUNDANCES}

We have used both IRAF and MOOG2 (Sneden 1973, as revised in 2002) to analyze the reduced spectra. Equivalent widths were measured with the splot task in IRAF for each star. The line list we used is given in the Appendix. We edited the Fe I and Fe II line lists to omit lines weaker than $5 \mathrm{~m} \AA$ that might have poorer quality measurements. Most of the line lists for Fe and the other elements are from Stephens (1999), Stephens \& Boesgaard (2002), Reddy et al. (2003), Kurucz (1995) and the NIST data site. Additions to those lists are described in the relevant parts of $\S 4$.

\subsection{Stellar Parameter Determination}

We have used the infra-red flux method (IRFM) to find $T_{\text {eff }}$. Table 2 gives the measured colors for our stars, primarily from 2MASS. Table 3 gives the temperatures derived from the color indices and the calibration of Casagrande et al. (2010).

With the exception of Sc II and Ba II, our abundances are not very sensitive to the value of $\log \mathrm{g}$. Inasmuch as we are dealing with stars in a cluster, we have used the relationship between log g and B-V compiled by Gray (1976) from 45 main-sequence eclipsing-binary stars. We have used the empirical relationship for microturbulent velocity derived by Edvardsson et al. (1993) with its dependency on $\log g$ and $T_{\text {eff }}$.

We determined values for $[\mathrm{Fe} / \mathrm{H}]$ from 50-55 lines of Fe I and 5-6 lines of Fe II. We found the mean $[\mathrm{Fe} / \mathrm{H}]$ by weighing Fe I and the Fe II results by the number of lines measured for each. In Table 4 we show these results for $[\mathrm{FeI} / \mathrm{H}]$ and $[\mathrm{FeII} / \mathrm{H}]$ along with the average deviation from the individual lines for each star. The final $<[\mathrm{Fe} / \mathrm{H}]>$ was $+0.117 \pm 0.039$. In the subsequent models for all the stars we used $[\mathrm{Fe} / \mathrm{H}]=0.12$. Table 5 gives the adopted model parameters for the 11 stars in this study.

\subsection{Abundance Determinations}

We used Kurucz (1993) model atmospheres and interpolated among his grid models to create a model atmosphere for each star. For Li we used the synth driver in MOOG to make synthetic spectra to determine the Li abundance. For the other elements we used measurements of equivalent widths and the abfind driver in MOOG, with the exception of

\footnotetext{
${ }^{2}$ http://www.as.utexas.edu/ chris/moog.html
} 
Ba II for which we include the hyperfine splitting (hfs) and use the blends driver. (The Appendix contains a table giving the wavelengths, excitation potentials, log gf values and measured equivalent widths of the lines used for the three stars shown in Figures $2-5$.) The final abundances are given in Table 6.

\subsection{Abundance Uncertainties}

We estimated errors due to uncertainties in the stellar parameters. Table 3 shows the agreement among the temperatures determined from the various color indices. The uncertainty in the mean ranges from 29 to $111 \mathrm{~K}$ with a mean of $56 \mathrm{~K}$, median of $50 \mathrm{~K}$. We chose $\pm 75 \mathrm{~K}$ as a conservative estimate of the uncertainty in $T_{\text {eff }}$. The average deviation from the linear relation from Gray between $\log g$ and $B-V$ is \pm 0.09 dex. Our choice of \pm 0.20 dex for the uncertainty in $\log \mathrm{g}$ is also conservative. Our mean $[\mathrm{Fe} / \mathrm{H}]$ of \pm 0.117 has a standard deviation of \pm 0.039 , so our use of \pm 0.10 in $[\mathrm{Fe} / \mathrm{H}]$ is again conservative. The Edvardsson et al. (1993) relation for microturbulent velocity has an rms scatter of $0.3 \mathrm{~km}$ $\mathrm{s}^{-1}$ based on 157 field stars; for cluster stars of virtually the same log g (4.38 - 4.43) the rms scatter is considerably less and we used $\pm 0.20 \mathrm{~km} \mathrm{~s}^{-1}$. Table 7 shows those abundance errors for Fe I. We added the errors in quadrature to estimate the total error due to the parameter uncertainties. In Table 8 we show the errors for the three representative stars shown in Figures 2-5 for the other elements.

\section{RESULTS}

\subsection{Iron}

The values for $[\mathrm{Fe} / \mathrm{H}]$ were given in Table 4 . These are plotted in Figure 6 where the errorbars shown are due to the parameter uncertainties for each star from Table 7 . We have derived the values of $[\mathrm{Fe} / \mathrm{H}]=+0.117 \pm 0.039$ (the sample standard deviation). Including the fact that there are 11 stars in our sample, the error is \pm 0.012 .

Our value for $[\mathrm{Fe} / \mathrm{H}]$ of +0.12 is in good agreement with that of An et al. (2007) who found $[\mathrm{Fe} / \mathrm{H}]=+0.11 \pm 0.03$ from four $\mathrm{G}$ dwarfs; there are two stars in common with this study, KW 23 and KW 58, which agree better than within the quoted errors. We do not agree with Pace et al. (2008) whose $[\mathrm{Fe} / \mathrm{H}]$ abundance from seven stars was supersolar at $+0.27 \pm 0.10$. Salaris et al. (2004) used +0.13 for $[\mathrm{Fe} / \mathrm{H}]$ and Carrera \& Pancino (2011) found $+0.16 \pm 0.05$ from three giants stars in Praesepe. 


\subsection{Lithium}

We have used MOOG with the synth driver to find Li abundances, A(Li) $=\log N(\mathrm{Li})$ +12.00 . The line list used for the Li synthesis is from King \& Hiltgen (1996). Figure 7 shows the abundance matches for two of our stars. For all 11 stars the syntheses were excellent fits to the data. Lithium abundances or upper limits have been determined by Soderblom et al. (1993) for 63 Praesepe stars with temperatures between 4970 and $6810 \mathrm{~K}$ from Li equivalent width measurements; these have been redetermined by them in Soderblom et al. (1995). Boesgaard \& Budge (1988) found Li abundances in seven additional Praesepe F dwarfs. In 1995 Balachandran recalibrated the temperature scale and excluded photometric binaries and double-lined spectroscopic binaries from those samples leaving 59 stars. Our synthesized spectral results agree well with those that Balachandran (1995) reexamined via equivalent width measurements. The mean difference in $\mathrm{A}(\mathrm{Li})$ is +0.07 dex and those differences are result from the temperature differences. Similarly, the agreement with the Soderblom et al. revised $\mathrm{Li}$ abundances is good with the mean difference in $\mathrm{A}(\mathrm{Li})=+0.04$. We have six stars in common with King \& Hiltgen (1996) where our mean A(Li) difference is +0.05 .

Soderblom et al. $(1993,1995)$ found that the low mass stars in the Praesepe cluster had higher Li abundances than the low mass stars in the Hyades with $\mathrm{T}_{\text {eff }}<5800 \mathrm{~K}$. Once Balachandran (1995) put the two clusters on the same temperature scale, that Li abundance difference disappeared. We show in our Figure 8 that Hyades and Praesepe have virtually the same Li abundances in the temperature range of our Praesepe observations: 5650 - 6000 K. Our Hyades Li abundances are taken from Boesgaard et al. (in preparation) re-evaluation of the Hyades Li abundances with the stellar parameters determined from the new Hipparcos calibration.

\subsection{Carbon and Oxygen}

Both $\mathrm{C}$ and $\mathrm{O}$ are formed from massive stars $\left(\gtrsim 10 \mathrm{M}_{\odot}\right)$ and - through core-collapse supernovae - have enriched the gas out of which the early generations of stars were formed. These, in turn, have added their own contributions to successive generations. The bulk of the $\mathrm{Fe}$ is formed in intermediate mass stars in supernovae type Ia, so the production of Fe lags behind that of $\mathrm{C}$ and O, e.g. Tinsley (1980), Wheeler et al. (1989). This leads to the expectation that $[\mathrm{C} / \mathrm{Fe}]$ and $[\mathrm{O} / \mathrm{Fe}]$ will be greater than zero in metal-poor stars. A recent study that included both $\mathrm{Fe}$ and $\mathrm{O}$ in 117 stars with $[\mathrm{Fe} / \mathrm{H}]$ from -0.5 to -3.5 by Boesgaard et al. (2011) shows a monotonic decrease in $[\mathrm{O} / \mathrm{Fe}]$ with $[\mathrm{Fe} / \mathrm{H}]$. At $[\mathrm{Fe} / \mathrm{H}]=-3.5$ the value for $[\mathrm{O} / \mathrm{Fe}]$ is +1.0 and by $[\mathrm{Fe} / \mathrm{H}]=-0.5$ the value for $[\mathrm{O} / \mathrm{Fe}]$ has declined to +0.2 . A similar 
trend was found by Boesgaard et al. (1999) and Israelian et al. (1998, 2001).

The seven lines of C I and three lines of O I (the O triplet) are all lines with high excitation potential. The gf values for C I are from Weise et al. (1996) and Reddy et al. (2003) while the O I triplet line gf values are from Weise et al. (1996). The C and O abundances in our Praesepe dwarfs are given in Table 6. The $\mathrm{O}$ abundances given there have been corrected for NLTE effects through the calculations of Takeda (2003). Rentzsch-Holm (1996) has studied the NLTE abundance corrections for C in stars with temperatures 7000 $-12,000 \mathrm{~K}, \log \mathrm{g}$ values of 3.5, 4.0, 4.5 and metallicities of $[\mathrm{M} / \mathrm{H}]$ of $-0.5,0.0,+0.5$, and +1.0. These trends indicate that for our solar-temperature stars with $\mathrm{C}$ I equivalent widths of $10-32 \mathrm{~m} \AA$, the NLTE effects are insignificant. We can compare our $\mathrm{C}$ and $\mathrm{O}$ abundances with those in field stars. We have selected the field stars from Edvardsson et al. (1993) for O comparisons and those in Takeda \& Honda (2005) for C and O. In both comparison samples we have restricted the range in $[\mathrm{Fe} / \mathrm{H}]$ to be 0.00 to +0.20 , enveloping our Praesepe range of +0.05 to +0.17 . In addition we have used the results from Reddy et al. $(2003,2006)$ for $\mathrm{C}$ in $\mathrm{F}$ and $\mathrm{G}$ dwarfs in the thin disk and the thin-thick disk in our metallicity and temperature range. None of the comparison stars is as young as Praesepe.

Figure 9 shows the ratio $[\mathrm{C} / \mathrm{Fe}]$ for the Praesepe stars in the top panel along with the Reddy et al. (2003, 2006) and Takeda \& Honda (2005) comparisons. By the solar age the excess of $\mathrm{C}$ over $\mathrm{Fe}$ is expected to have disappeared. In the young Praesepe cluster (age $\sim 0.7$ $\mathrm{Gyr}$ ) the value we find for $[\mathrm{C} / \mathrm{Fe}]$ of $-0.14 \pm 0.07$ could indicate the continued decrease in $[\mathrm{C} / \mathrm{Fe}]$ with time.

The lower panel of Figure 9 shows $[\mathrm{O} / \mathrm{Fe}]$ as corrected for NLTE effects for Praesepe and the two comparison samples of Edvardsson et al. (1993) and Takeda \& Honda (2005). (Edvardsson et al. (1993) did not do a correction for NLTE effects, but rather calibrated their abundances found from the $\mathrm{O} I$ triplet alone to the abundances found in those stars where they had results from both the [O I] line at $6300 \AA$ and the O I triplet.) As is the case for $[\mathrm{C} / \mathrm{Fe}]$, we find that $\mathrm{O}$ has decreased relative to $\mathrm{Fe}$ and the mean value of $[\mathrm{O} / \mathrm{Fe}]$ is $-0.15 \pm 0.09$. The cluster stars have $\mathrm{O}$ abundances typically below the field star sample.

\subsection{Alpha-Elements: $\mathrm{Mg}, \mathrm{Si}, \mathrm{Ca}, \mathrm{Ti}$}

For the abundance determinations we have used two lines of Mg I, 11 lines of Si I, 10 lines of Ca I, and 11 lines of Ti I as listed in the Appendix. The alpha-element abundances are given for each star in Table 6. Their ratios normalized to Fe are shown in Figures 10 and 11 along with the mean values for $[\mathrm{X} / \mathrm{Fe}]$ and $[\mathrm{Fe} / \mathrm{H}]$ for Praesepe. The comparison samples 
are from Edvardsson et al. (1993) and Reddy et al. (2003, 2006). The field stars, except for $\mathrm{Ca}$, show a larger range in abundance than do the Praesepe stars. And the Praesepe stars, again except for $\mathrm{Ca}$, have lower values of $[\mathrm{X} / \mathrm{Fe}]$ than the field stars. The alpha-element abundances relative to Fe (relative to solar) tend to decrease with age reaching solar at the age of the Sun. The Praesepe stars are all younger than the field star sample and do show solar abundances. The alpha ratios relative to $\mathrm{Fe}$ are all close to solar: $[\mathrm{Mg} / \mathrm{Fe}]=-0.003$ $\pm 0.040 ;[\mathrm{Ti} / \mathrm{Fe}]=-0.035 \pm 0.062 ;[\mathrm{Ca} / \mathrm{Fe}]=-0.006 \pm 0.049 ;[\mathrm{Si} / \mathrm{Fe}]=-0.005 \pm 0.031$. The mean ratio, $[<\alpha>/ \mathrm{Fe}]$, is $-0.012 \pm 0.015$.

According to Tsujimoto et al. (1995) the contribution of SNe Ia to the solar abundances is only $\sim 1 \%$ for $\mathrm{Mg}, \sim 25 \%$ for $\mathrm{Ca}$, and $\sim 17 \%$ for $\mathrm{Si}$. With the exception of $\mathrm{Mg}$, both $\mathrm{SNe}$ Ia and SN II have contributed to the alpha-elements in the Praesepe cluster.

\subsection{Fe-Peak Elements: $\mathrm{Cr}$ and $\mathrm{Ni}$}

In addition to the 55 lines of Fe I and six lines of Fe II, we have measured nine lines of $\mathrm{Cr} \mathrm{I}$ and 26 lines of $\mathrm{Ni}$ I. Abundances for $\mathrm{Cr}$ and $\mathrm{Ni}$ are given in Table 6 for each star along with the mean abundances relative to $\mathrm{Fe}$ and the standard deviation of the mean. Figure 12 shows the results as a function of $[\mathrm{Fe} / \mathrm{H}]$ with the comparison samples of field stars. Bergemann \& Cescutti (2010) studied the effects of NLTE on Cr I in the Sun and metal-poor stars. Our Cr abundances have been corrected for the overionization effect on Cr I using the solar value of $\log \epsilon(\mathrm{Cr} \text { II })_{\odot}=5.77$ from Sobeck et al. (2007). The Praesepe cluster mean, $[\mathrm{Cr} / \mathrm{Fe}]=0.003 \pm 0.031$, is the solar value and in agreement with the field star

sample. According to Clayton (2003) both SN II and SN Ia produce Cr/Fe ratios that are roughly solar.

The mean value we find for $[\mathrm{Ni} / \mathrm{Fe}]$ is also similar to the solar value at $-0.028 \pm 0.027$. The abundances are comparable to the field stars from Reddy et al. $(2003,2006)$, but seem lower than the bulk of the Edvardsson et al. (1993) field stars of comparable metallicity. Our two iron-peak elements behave as Fe does.

\section{6. n-Capture Elements: $\mathrm{Y}$ and $\mathrm{Ba}$}

We have determined abundances for the two n-capture elements, $\mathrm{Y}$ and $\mathrm{Ba}$, which are dominated by the s-process at different s-process peaks. For Y we have measured two lines of Y I and three lines of Y II, but not all lines were measurable in most of the stars; we also measured these lines in our lunar spectrum. The gf values for Y II are from Hannaford et 
al. (1982). We then normalized our stellar Y abundance to our lunar/solar $\mathrm{Y}$ to get $[\mathrm{Y} / \mathrm{H}]$ for the Praesepe stars. For Ba we had one line of Ba II at 5853.7 $\AA$ for which we included the hyperfine structure in our analysis. The results for $[\mathrm{Y} / \mathrm{Fe}]$ and $[\mathrm{Ba} / \mathrm{Fe}]$ are given in Table 6. Figure 13 shows the results for $[\mathrm{Y} / \mathrm{Fe}]$ (upper panel) and [Ba/Fe] (lower panel) with the field star comparison samples. The field stars show a large range $( \pm 0.20)$ in both elements; our $[\mathrm{Y} / \mathrm{Fe}]$ vales are solar near the middle of the comparison stars. Our $[\mathrm{Ba} / \mathrm{Fe}]$ are somewhat higher than solar at $+0.11 \pm 0.04$ and near the top of the field star results. This is consistent with the results of D'Orazi et al. (2009) who found that $[\mathrm{Ba} / \mathrm{Fe}]$ increases in open clusters with younger ages. They suggest that the enhancement of $[\mathrm{Ba} / \mathrm{Fe}]$ would come from low mass stars $\left(\lesssim 1.5 \mathrm{M}_{\odot}\right)$.

\subsection{Other Elements: $\mathrm{Na}, \mathrm{Al}, \mathrm{Sc}, \mathrm{V}$}

We also found abundances for some other elements of interest. We have measured equivalent widths of two lines of $\mathrm{Na} \mathrm{I}$, one $\mathrm{Al}$ I line, two lines of Sc II, and two lines of $\mathrm{V}$ I. (Reddy et al. (2003) showed in test calculations that the effects of hyperfine splitting on the lines they used of V I and Sc II have virtually no effect on the abundances derived; we used those same lines.) Figure 14 shows the results for $\mathrm{Na}$ and $\mathrm{Al}$. The value for $[\mathrm{Na} / \mathrm{Fe}]$ and $[\mathrm{Al} / \mathrm{Fe}]$ are solar at $-0.011 \pm 0.032$ and $0.004 \pm 0.038$, respectively, and are in good agreement with the field star sample. See $\S 5$ for an interesting comparison with $\mathrm{Na}$ in the Praesepe giants.

In Figure 15 we show the results for $\mathrm{V}$ and $\mathrm{Sc}$. The ratios of $[\mathrm{V} / \mathrm{Fe}]$ and $[\mathrm{Sc} / \mathrm{Fe}]$ are somewhat greater than solar at $+0.035 \pm 0.053$ and $0.036 \pm 0.024$, respectively, but they are basically solar within the errors. The field stars from the Reddy papers are similar, but, as expected, they have greater scatter compared to the cluster stars.

\section{COMPARISON WITH PRAESEPE GIANTS}

Carrera \& Pancino (2011) determined abundances of many elements in three red giants in Praesepe. We can compare our results for solar-temperature dwarf stars with theirs for the red giants. Table 9 and Figure 17 show those comparisons. Our values for $[\mathrm{Fe} / \mathrm{H}]$ are in good agreement within our 1 sigma errors.

Their results for $[\mathrm{Na} / \mathrm{Fe}]$ show an enhancement to $+0.25 \pm 0.06$ from our solar value $([\mathrm{Na} / \mathrm{Fe}]=-0.01 \pm 0.03)$. Our two studies have used the same $\mathrm{Na}$ lines and the same gf values. The lines selected are weak $\mathrm{Na}$ lines and expected to have only minor NLTE 
corrections, e.g. Asplund (2005). Each of their three red giants shows the enhancement of $[\mathrm{Na} / \mathrm{Fe}]:+0.23,+0.30,+0.18$. Such a Na-enhancement could be the result of protoncapture nucleosynthesis in the $\mathrm{Ne} \rightarrow \mathrm{Na}$ cycling with dredge-up into the atmospheres of the red giants. Enhanced $\mathrm{Na}$ and an anti-correlation between $\mathrm{Na}$ and $\mathrm{O}$ has been discovered recently in evolved red giants in the populous old open cluster, NGC 6791, by Geisler et al. (2012). There are too few stars in Praesepe that have evolved to become red giants to detect any such effect in Praesepe. All three giants studied by Carrera \& Pancino (2011) do have low $[\mathrm{O} / \mathrm{Fe}]$ at $-0.11 \pm 0.03$.

For the alpha elements, $\mathrm{Si}, \mathrm{Ca}$, and $\mathrm{Ti}$, the agreement is very good and close to solar in both dwarfs and giants. However, Carrera \& Pancino (2011) found $[\mathrm{Mg} / \mathrm{Fe}]$ is enhanced to +0.27 in the giants. Our value for the dwarf stars is solar. Their three giants give similar values for $[\mathrm{Mg} / \mathrm{Fe}]:+0.22,+0.27,+0.31$. Our two studies have measured different lines of $\mathrm{Mg}$ I. Two of their four lines are quite strong at more than $100 \mathrm{~m} \AA$. It is not expected that $\mathrm{Mg}$ would be increased in the giants, rather the $\mathrm{Mg} \rightarrow \mathrm{Al}$ cycling would result in a decrease in Mg. Yong et al. (2003) find a positive correlation between $\mathrm{Al}$ and $\mathrm{Mg}$ for some giant stars in the globular cluster, NGC 6752, apparently due to an increase in ${ }^{26} \mathrm{Mg}$ (see their Figure 12). It might be interesting to determine the $\mathrm{Mg}$ isotope ratios in those giant stars. On the other hand, the $[\mathrm{Al} / \mathrm{Fe}]$ abundances in both the dwarfs and the giants in Praesepe are solar.

Our Ba abundances are from the Ba II line at $5853 \AA$ in which we included the hyperfine structure of that line. Our mean cluster abundance for $[\mathrm{Ba} / \mathrm{Fe}]$ is $+0.11 \pm 0.04$ for the main sequence stars which is consistent with the findings of D'Orazi et al. (2009) discussed in §4.6. Carrera \& Pancino (2011) found $[\mathrm{Ba} / \mathrm{Fe}]$ is $+0.33 \pm 0.05$ for their three giants. This could be evidence for $\mathrm{n}$-capture-enriched $\mathrm{Ba}$ in the giants. However, apparently Carrera \& Pancino (2011) did not use the hyperfine splitting (hfs) in their Ba analysis. In order to determine the effect of the hfs on the Ba abundance, we determined Ba abundances without including the hfs for our 11 dwarf stars. We find that including the hfs reduces the Ba abundance by 0.03 dex. If the correction is similar for giants, then $[\mathrm{Ba} / \mathrm{Fe}]$ would be +0.30 in those three giants; this is still an increase over $[\mathrm{Ba} / \mathrm{Fe}]$ in the dwarfs.

\section{SUMMARY AND CONCLUSIONS}

We have analyzed high-resolution, high S/N spectra of 11 solar-temperature main sequence stars in the Praesepe open cluster obtained with Keck I + HIRES. The spectra cover a region from $5730-8140 \AA$. We have determined stellar temperatures from the infrared flux method and found Fe abundances from some 55 Fe I lines and 6 Fe II lines in each star. The $[\mathrm{Fe} / \mathrm{H}]$ agreement between the $\mathrm{Fe} \mathrm{I}$ and Fe II lines is excellent and the cluster mean is 
$[\mathrm{Fe} / \mathrm{H}]=0.12 \pm 0.04( \pm 0.01)$

We determined $\mathrm{Li}$ abundances by the spectral synthesis method and found them to track the Hyades Li abundances very well showing a steady decline from $6000 \mathrm{~K}$ to $5650 \mathrm{~K}$; the decline is due to increasing $\mathrm{Li}$ depletion at decreasing temperatures. We find that $[\mathrm{C} / \mathrm{Fe}]$ and $[\mathrm{O} / \mathrm{Fe}]$ to be $-0.14 \pm 0.07$ and $-0.15 \pm 0.09$, respectively. These values are similar, but somewhat lower than the field star samples and we interpret the lower values to be due to the younger age of Praesepe relative to the field stars. This follows the steady decline in $[\mathrm{C} / \mathrm{Fe}]$ and $[\mathrm{O} / \mathrm{Fe}]$ over time from the early excess of $\mathrm{C}$ and $\mathrm{O}$ over $\mathrm{Fe}$ as produced by the most massive stars and SN II followed by the rise in Fe from SN Ia. All the $\alpha$-elements, $[\mathrm{Mg} / \mathrm{Fe}],[\mathrm{Si} / \mathrm{Fe}],[\mathrm{Ca} / \mathrm{Fe}]$, and $[\mathrm{Ti} / \mathrm{Fe}]$ are solar at $0.00 \pm 0.04,-0.01 \pm 0.03,-0.01 \pm 0.05$, and $-0.04 \pm 0.06$, respectively. As is the case for $[\mathrm{C} / \mathrm{Fe}]$ and $[\mathrm{O} / \mathrm{Fe}]$, the $\alpha$-elements, $\mathrm{Mg}, \mathrm{Ti}$, and $\mathrm{Si}$, are somewhat lower relative to Fe compared to the field stars. For $[\mathrm{Ca} / \mathrm{Fe}]$ the field star sample is also solar and has less spread in the values.

The abundances of the Fe-peak elements, $[\mathrm{Cr} / \mathrm{Fe}]$ and $[\mathrm{Ni} / \mathrm{Fe}]$, were found to track $\mathrm{Fe}$ and are basically solar at $0.00 \pm 0.03$ and $-0.03 \pm 0.03$, respectively. The n-capture element $\mathrm{Y}$ from the first s-process peak was found to be solar at $[\mathrm{Y} / \mathrm{Fe}]=0.01 \pm 0.08$ and in good agreement with the field stars. The n-capture element $\mathrm{Ba}$ from the second s-process peak was found to be enhanced relative to solar and relative to the field stars with $[\mathrm{Ba} / \mathrm{Fe}]=$ $+0.11 \pm 0.04$. For the Praesepe stars $[\mathrm{V} / \mathrm{Fe}],[\mathrm{Sc} / \mathrm{Fe}],[\mathrm{Na} / \mathrm{Fe}]$, and $[\mathrm{Al} / \mathrm{Fe}]$ are essentially solar at $0.04 \pm 0.05,0.04 \pm 0.02,-0.01 \pm 0.03$, and $0.00 \pm 0.04$, respectively. Both $[\mathrm{Na} / \mathrm{Fe}]$ and $[\mathrm{Al} / \mathrm{Fe}]$ are somewhat lower than the field stars and have less spread in the values, as expected in a cluster of stars of common origin.

The comparison with the composition of the giant stars in Praesepe yielded some interesting differences for $\mathrm{Na}, \mathrm{Mg}$, and $\mathrm{Ba}$. The three red giants studied by Carrera \& Pancino (2011) show an enhancement in $[\mathrm{Na} / \mathrm{Fe}]$ of +0.26 compared to our dwarf stars. The enhancement of $\mathrm{Na}$ might be caused by the proton-capture nucleosynthesis in the $\mathrm{Ne} \rightarrow \mathrm{Na}$ cycling in the interior and subsequent dredge-up in the giant stars. For the $\alpha$-elements Si, $\mathrm{Ca}$, and $\mathrm{Ti}$ the dwarfs and the giants are similar within the errors. However, they found an enhancement in $[\mathrm{Mg} / \mathrm{Fe}]$ in the giants of +0.27 . If there is $\mathrm{Mg} \rightarrow \mathrm{Al}$ cycling, that would lead to a decrease in $[\mathrm{Mg} / \mathrm{Fe}]$ and an increase in $[\mathrm{Al} / \mathrm{Fe}]$; for both dwarfs and giants $[\mathrm{Al} / \mathrm{Fe}]$ $=0.00$ with similar uncertainties $\sim \pm 0.04$. It may be important to measure the $\mathrm{Mg}$ isotopes in the giants in case the increase in $\mathrm{Mg}$ is due to an increase in ${ }^{26} \mathrm{Mg}$. Barium appears to be enhanced in the giants as well. We find $[\mathrm{Ba} / \mathrm{Fe}]=+0.11 \pm 0.04$ for the dwarf stars while they derive $+0.33 \pm 0.05$. The giants may be enriched by the s-process n-capture. However, there is no apparent enrichment of $[\mathrm{Y} / \mathrm{Fe}]$ in the giants. 
We would like to thank John Lakatos for his help with the observing and data reduction and $\mathrm{Hai} \mathrm{Fu}$ for his IRAF routine to expedite equivalent width measurements. We acknowledge support from NSF through grant AST 05-05899 to A.M.B. 


\section{REFERENCES}

An, D., Terndrup, D.M., Pinsonneault, M.H., Paulson, D., Hanson, R.B. \& Stauffer, J. 2007, ApJ, 655, 233-260

Asplund, M. 2005, ARAA, 43, 481

Balachandran, S. 1995, ApJ, 446, 203

Barrado y Navascues, D., Stauffer, J. \& Randich, S. 1998, ApJ, 506, 347-359

Bergemann, M. \& Cescutti, G. 2010, A\&A, 522, A9

Boesgaard, A.M. 1989, ApJ, 336, 798

Boesgaard, A.M. \& Budge, K.G. 1988, ApJ, 332, 410

Boesgaard, A. M., King, J. R., Deliyannis, C. P. \& Vogt, S. S. 1999, AJ, 117, 492

Carrera, R. \& Pancino, E. 2011, A\&A, 535, A30

Casagrande, L., Ramrez, I., Melndez, J., Bessell, M. \& Asplund, M. 2010 A\&A, 512, 54

Clayton, D. 2003 "Handbook of Isotopes in the Cosmos,"

D’Orazi, V., Magrini, L., Randich, S., Galli, D., Busso, M. \& Sestito, P. 2009, ApJ, 693, L31.

Edvardsson, B., Andersen, J., Gustafsson, B., Lambert, D.L., Nissen, P.E. \& Tomkin, J. 1993, A\&A, 275, 101-150

Eggen, O.J. 1992, AJ, 104, 1482

Franciosini, E., Randich, S. \& Pallavicini, R. 2003, A\&A, 405, 551-561

Friel, E.D. \& Boesgaard, A.M. 1992, ApJ, 387, 170

Geisler, D., Villanova, S., Carraro, G., Pilachowski, C., Cummings, J., Johnson, C. I. \& Bresolin, F. 2012 A\&A, 756, 40

Gratton, R. 2000, in "Stellar Clusters and Associations" eds. R. Pallavicini, G. Micela \& S. Sciortino (San Francisco: A.S.P) ASPC, 198, 225

Gray, D.F. 1976 in "The Observation and Analysis of Stellar Photospheres" (New York: John Wiley \& Sons) p. 389

Hannaford, P, Lowe, R.M., Grevesse, N., Biemont, E. \& Whaling, W. 1982, ApJ, 261, 736

Holland, K., Jameson, R.F., Hodgkin, S., Davies, M.B. \& Pinfield, D. 2000, MNRAS, 319, 956

Israelian, G., Garca Lpez, R.J. \& Rebolo, R. 1998, ApJ, 507, 885 
Israelian, G., Rebolo, R., Garca Lpez, R.J., Bonifacio, P., Molaro, P. Basri, G. \& Shchukina, N. 2001, ApJ, 551, 833

Johnson, H.L. 1952, ApJ, 116, 640

Jones, B.F. \& Cudworth, K.M. 1983, AJ, 88, 215

Jones, B.F. \& Stauffer, J.R. 1991, AJ102, 1080

King, J.R. \& Hiltgen, D. 1996, PASP, 108, 246

Kraft, R.P., Sneden, C., Smith, G.H., Shetrone, M.D., Langer, G. E. \& Pilachowski, C.A. 1997 AJ, 113, 279

Kurucz, R. L. 1993, CD-ROM 13 (Cambridge: Smithsonian Astrophys. Obs.)

Kurucz, R.L. 1995, CD-ROM No. 23. Cambridge, Mass.: Smithsonian Astrophysical Observatory

Magrini, L., Sestito, P., Randich, S. \& Galli, D. 2009, A\&A, 494, 95-108

Mendoza, E.E. 1967, Bol. Obs. Tonantzintla Tacubaya, 4, 149

Mermilliod, J.C. \& Mayor, M. 1999, A\&A, 352, 479

Pace, G., Pasquini, L. \& Francois, P. 2008, A\&A, 489, 403-412

Palous, J., Ruprecht, J., Dluzhnevskaya, O.B. \& Piskunov, T. 1977, A\&A, 61, 27-37

Randich, S. \& Schmitt, J.H.M.M. 1995, A\&A, 298, 115-132

Reddy, B., Lambert, D.L. \& Allende Prieto, C. 2006, MNRAS, 367, 1329

Reddy, B., Tompkin, J., Lambert, D.L. \& Allende Prieto, C. 2003, MNRAS, 340, 304

Rentzsch-Holm, I. 1996, A\&A, 312, 966

Salaris, M., Weiss, A. \& Percival, S.M. 2004, A\&A, 414, 163-174

Sneden, C. 1973, PhD thesis, Univ. of Texas, Austin

Sneden, C., Kraft, R.P., Prosser, C.F. \& Langer, G. E. 1991, AJ, 102, 2001

Sobeck, J.S., Lawler, J.E. \& Sneden, C. 2007, ApJ, 667, 1267

Soderblom, D.R., Fedele, S.B., Jones, B.F., Stauffer, J. R. \& Prosser, C.F. 1993, AJ, 106, 1080

Soderblom, D.R., Fedele, S.B., Jones, B.F., Stauffer, J. R. \& Prosser, C.F. 1995, AJ, 109, 1402

Stern, R.A., Schmitt, J.H.M.M. \& Kahabka, P.T. 1995, ApJ, 448, 683-704

Stephens, A. 1999, AJ, 117, 1771 
Stephens, A. \& Boesgaard, A.M. 2002, AJ, 123, 1647

Takeda, Y. 2003, A\&A, 402, 343

Takeda, Y. \& Honda, S. 2005, PASJ, 57, 65

Tinsley, B.M. 1980, Fund. Cosmic Phys. 5, 287

Tsujimoto, T., Yoshii, Y., Nomoto, K. \& Shigeyama, T. 1995, A\&A, 302, 704

Vogt, S. S., Allen, S. L., Bigelow, B. C., Bresee, L., Brown, B., Cantrall, T., Conrad, A., Couture, M., Delaney, C., Epps, H. W., and 17 coauthors 1994, SPIE, 2198, 362

Weise, W.L., Fuhr, J.R., \& Deters, T.M. (1996), in Atomic Transition Probabilities of Carbon, Nitrogen, and Oxygen: A Critical Data Compilation. NIST, QC 453 Wheeler, J. C., Sneden, C. \& Truran, J.W., Jr. 1989, ARA\&A, 27, 279

Yong, D., Grundahl, F., Lambert, D.L., Nissen, P.E. \& Shetrone, M.D. 2003, A\&A, 402, 985 
Table 1. Keck/HIRES Observations of Praesepe

\begin{tabular}{ccccccc}
\hline \hline Star & V & B-V & ref. & $\begin{array}{c}\text { Night } \\
\text { UT }\end{array}$ & $\begin{array}{c}\text { Exp. Time } \\
(\min )\end{array}$ & $\begin{array}{c}\text { Total } \\
\text { S/N }\end{array}$ \\
\hline KW 23 & 11.29 & 0.710 & 1 & 11 Jan 2003 & 25 & 149 \\
KW 30 & 11.40 & 0.730 & 1 & 11 Jan 2003 & 25 & 141 \\
KW 58 & 11.26 & 0.671 & 2 & 11 Feb 2003 & 25 & 159 \\
KW 181 & 10.47 & 0.588 & 2 & 11 Jan 2003 & 10 & 93 \\
KW 208 & 10.66 & 0.583 & 2 & 11 Jan 2003 & 14 & 153 \\
KW 288 & 10.69 & 0.593 & 2 & 11 Feb 2003 & 15 & 150 \\
KW 301 & 11.17 & 0.655 & 2 & 11 Feb 2003 & 25 & 155 \\
KW 335 & 11.03 & 0.651 & 2 & 11 Jan 2003 & 20 & 156 \\
KW 399 & 11.93 & 0.624 & 2 & 11 Jan 2003 & 20 & 166 \\
KW 432 & 11.05 & 0.646 & 2 & 11 Feb 2003 & 20 & 143 \\
KW 508 & 10.77 & 0.594 & 2 & 11 Jan 2003 & 20 & 159 \\
\hline
\end{tabular}

${ }^{1} 1=$ Mendoza (1967), $2=$ Johnson (1952) 
Table 2. Colors

\begin{tabular}{cccccccccc}
\hline \hline Star & B & $\mathrm{V}$ & $\mathrm{J}$ & $\mathrm{H}$ & $\mathrm{K}$ & $\mathrm{V}_{c}$ & $\mathrm{~V}-\mathrm{R}_{c}$ & $\mathrm{R}_{c}-\mathrm{I}_{c}$ & $\mathrm{~V}-\mathrm{I}_{c}$ \\
\hline KW 23 & 12.00 & 11.29 & 10.075 & 9.780 & 9.686 & $\ldots$ & $\ldots$ & $\ldots$ & $\ldots$ \\
KW 30 & 12.13 & 11.40 & 10.187 & 9.898 & 9.803 & $\ldots$ & $\ldots$ & $\ldots$ & $\ldots$ \\
KW 58 & 11.93 & 11.26 & 10.079 & 9.791 & 9.689 & $\ldots$ & $\ldots$ & $\ldots$ & $\ldots$ \\
KW 181 & 11.06 & 10.47 & 9.357 & 9.088 & 8.997 & 10.488 & 0.339 & 0.325 & 0.664 \\
KW 208 & 11.24 & 10.66 & 9.565 & 9.357 & 9.259 & $\ldots$ & $\ldots$ & $\ldots$ & $\ldots$ \\
KW 288 & 11.28 & 10.69 & 9.640 & 9.401 & 9.336 & 10.698 & 0.333 & 0.308 & 0.641 \\
KW 301 & 11.83 & 11.17 & 10.012 & 9.698 & 9.655 & $\ldots$ & $\ldots$ & $\ldots$ & $\ldots$ \\
KW 335 & 11.68 & 11.03 & 9.864 & 9.588 & 9.507 & $\ldots$ & $\ldots$ & $\ldots$ & $\ldots$ \\
KW 399 & 11.55 & 10.93 & 9.812 & 9.549 & 9.469 & $\ldots$ & $\ldots$ & $\ldots$ & $\ldots$ \\
KW 432 & 11.70 & 11.05 & 9.869 & 9.627 & 9.544 & $\ldots$ & $\ldots$ & $\ldots$ & $\ldots$ \\
KW 508 & 11.36 & 10.77 & 9.659 & 9.416 & 9.359 & 10.761 & 0.334 & 0.326 & 0.660 \\
\hline
\end{tabular}


Table 3. Temperatures

\begin{tabular}{ccccccccr}
\hline \hline Star & $\mathrm{T}_{B-V}$ & $\mathrm{~T}_{V-J}$ & $\mathrm{~T}_{V-H}$ & $\mathrm{~T}_{V-I c}$ & $\mathrm{~T}_{V-R c}$ & $\mathrm{~T}_{R c-I c}$ & mean $\mathrm{T}_{\text {eff }}$ & $\sigma$ \\
\hline KW 23 & 5615 & 5703 & 5772 & $\ldots$ & $\ldots$ & $\ldots$ & 5697 & \pm 79 \\
KW 30 & 5554 & 5708 & 5770 & $\ldots$ & $\ldots$ & $\ldots$ & 5677 & \pm 111 \\
KW 58 & 5697 & 5771 & 5813 & $\ldots$ & $\ldots$ & $\ldots$ & 5761 & \pm 59 \\
KW 181 & 5922 & 5910 & 5954 & 5868 & 5918 & 5824 & 5899 & \pm 46 \\
KW 208 & 5978 & 5950 & 6065 & $\ldots$ & $\ldots$ & $\ldots$ & 5997 & \pm 60 \\
KW 288 & 6006 & 6050 & 6078 & 5958 & 5954 & 5988 & 6006 & \pm 50 \\
KW 301 & 5776 & 5815 & 5836 & $\ldots$ & $\ldots$ & $\ldots$ & 5809 & \pm 30 \\
KW 335 & 5861 & 5800 & 5867 & $\ldots$ & $\ldots$ & $\ldots$ & 5842 & \pm 37 \\
KW 399 & 5874 & 5901 & 5932 & $\ldots$ & $\ldots$ & $\ldots$ & 5903 & \pm 29 \\
KW 432 & 5841 & 5768 & 5914 & $\ldots$ & $\ldots$ & $\ldots$ & 5841 & \pm 73 \\
KW 508 & 5958 & 5919 & 5951 & 5887 & 5943 & 5831 & 5915 & \pm 48 \\
\hline
\end{tabular}


Table 4. $\quad[\mathrm{Fe} / \mathrm{H}]$ Abundances from Fe I and Fe II.

\begin{tabular}{lccccccc}
\hline \hline \multicolumn{1}{c}{ Star } & {$[\mathrm{FeI} / \mathrm{H}]$} & $\sigma$ & num. & {$[\mathrm{FeII} / \mathrm{H}]$} & $\sigma$ & num. & mean $[\mathrm{Fe} / \mathrm{H}]$ \\
\hline KW 23 & 0.15 & \pm 0.08 & 54 & 0.09 & \pm 0.11 & 6 & 0.144 \\
KW 30 & 0.12 & \pm 0.08 & 55 & 0.08 & \pm 0.09 & 6 & 0.116 \\
KW 58 & 0.11 & \pm 0.09 & 55 & 0.10 & \pm 0.06 & 6 & 0.109 \\
KW 181 & 0.13 & \pm 0.10 & 50 & 0.29 & \pm 0.09 & 5 & 0.145 \\
KW 208 & 0.10 & \pm 0.10 & 55 & 0.07 & \pm 0.09 & 6 & 0.097 \\
KW 288 & 0.08 & \pm 0.09 & 54 & 0.08 & \pm 0.07 & 6 & 0.080 \\
KW 301 & 0.17 & \pm 0.09 & 55 & 0.12 & \pm 0.09 & 6 & 0.165 \\
KW 335 & 0.14 & \pm 0.09 & 55 & 0.08 & \pm 0.09 & 6 & 0.134 \\
KW 399 & 0.08 & \pm 0.09 & 55 & 0.05 & \pm 0.09 & 6 & 0.077 \\
KW 432 & 0.18 & \pm 0.09 & 55 & 0.10 & \pm 0.09 & 6 & 0.172 \\
KW 508 & 0.04 & \pm 0.10 & 55 & 0.13 & \pm 0.06 & 6 & 0.048 \\
\hline
\end{tabular}


Table 5. Parameters for the Stellar Models

\begin{tabular}{lcccc}
\multicolumn{1}{c}{ Star } & $T_{\text {eff }}$ & $\log \mathrm{g}$ & {$[\mathrm{Fe} / \mathrm{H}]$} & $\xi$ \\
\hline KW 23 & 5699 & 4.43 & 0.12 & 1.10 \\
KW 30 & 5675 & 4.44 & 0.12 & 1.07 \\
KW 58 & 5761 & 4.42 & 0.12 & 1.16 \\
KW 181 & 5899 & 4.39 & 0.12 & 1.31 \\
KW 208 & 5997 & 4.38 & 0.12 & 1.40 \\
KW 288 & 6006 & 4.38 & 0.12 & 1.41 \\
KW 301 & 5809 & 4.41 & 0.12 & 1.21 \\
KW 335 & 5842 & 4.40 & 0.12 & 1.26 \\
KW 399 & 5903 & 4.40 & 0.12 & 1.31 \\
KW 432 & 5841 & 4.40 & 0.12 & 1.25 \\
KW 508 & 5915 & 4.39 & 0.12 & 1.33 \\
\hline
\end{tabular}


Table 6. Chemical Abundances for Praesepe Stars

\begin{tabular}{lrrrrrrrrrrrrrrrrr}
\hline \hline & KW 23 & KW 30 & KW 58 & KW 181 & KW 208 & KW 288 & KW 301 & KW 335 & KW 399 & KW 432 & KW 508 mean & \multicolumn{1}{c}{$\sigma$} \\
\hline$[\mathrm{Fe} / \mathrm{H}]$ & 0.144 & 0.116 & 0.109 & 0.145 & 0.097 & 0.080 & 0.163 & 0.134 & 0.077 & 0.172 & 0.048 & 0.117 & 0.039 \\
$\mathrm{~A}(\mathrm{Li})$ & 1.88 & 2.02 & 2.22 & 2.47 & 2.80 & 2.75 & 2.42 & 2.54 & 2.64 & 2.45 & 2.68 & $\ldots$ & $\ldots$ \\
{$[\mathrm{C} / \mathrm{Fe}]$} & -0.14 & -0.04 & -0.04 & -0.01 & -0.12 & -0.14 & -0.12 & -0.12 & -0.11 & -0.12 & -0.03 & -0.090 & 0.048 \\
{$[\mathrm{O} / \mathrm{Fe}] \mathrm{n}$} & -0.19 & -0.10 & -0.19 & -0.01 & -0.18 & -0.13 & -0.18 & -0.14 & -0.23 & -0.28 & 0.02 & -0.146 & 0.089 \\
{$[\mathrm{Na} / \mathrm{Fe}]$} & -0.05 & -0.06 & -0.04 & -0.01 & -0.03 & 0.03 & 0.01 & -0.01 & 0.00 & 0.00 & 0.04 & -0.011 & 0.032 \\
{$[\mathrm{Mg} / \mathrm{Fe}]$} & -0.04 & -0.02 & -0.02 & -0.07 & -0.03 & 0.04 & 0.01 & 0.02 & 0.02 & -0.01 & 0.07 & -0.003 & 0.040 \\
{$[\mathrm{Al} / \mathrm{Fe}]$} & -0.01 & -0.03 & 0.01 & 0.05 & -0.02 & 0.04 & -0.07 & -0.01 & 0.01 & 0.01 & 0.06 & 0.004 & 0.038 \\
{$[\mathrm{Si} / \mathrm{Fe}]$} & -0.04 & -0.02 & -0.01 & -0.04 & 0.03 & 0.02 & -0.02 & 0.01 & 0.01 & -0.04 & 0.05 & -0.005 & 0.031 \\
{$[\mathrm{Ca} / \mathrm{Fe}]$} & 0.06 & 0.00 & 0.01 & -0.01 & -0.06 & -0.02 & 0.00 & 0.04 & -0.01 & 0.06 & -0.04 & -0.006 & 0.049 \\
{$[\mathrm{Sc} / \mathrm{Fe}]$} & 0.05 & 0.03 & 0.01 & 0.01 & 0.04 & 0.03 & 0.07 & 0.00 & 0.03 & 0.07 & 0.06 & 0.036 & 0.024 \\
{$[\mathrm{Ti} / \mathrm{Fe}]$} & -0.03 & 0.02 & 0.02 & -0.06 & -0.11 & -0.08 & -0.06 & -0.04 & -0.07 & 0.10 & -0.05 & -0.033 & 0.059 \\
{$[\mathrm{~V} / \mathrm{Fe}]$} & -0.01 & 0.03 & 0.02 & 0.04 & 0.06 & 0.11 & 0.02 & 0.07 & 0.04 & 0.05 & -0.02 & 0.037 & 0.036 \\
{$[\mathrm{Cr} / \mathrm{Fe}]$} & 0.01 & -0.01 & -0.02 & 0.06 & 0.02 & 0.00 & -0.03 & 0.00 & 0.01 & 0.04 & -0.05 & 0.003 & 0.031 \\
{$[\mathrm{Ni} / \mathrm{Fe}]$} & -0.01 & -0.05 & -0.01 & -0.07 & -0.03 & -0.03 & 0.01 & -0.02 & -0.05 & 0.01 & -0.06 & -0.028 & 0.027 \\
{$[\mathrm{Y} / \mathrm{Fe}]$} & 0.15 & 0.08 & 0.06 & -0.07 & -0.11 & -0.08 & 0.05 & -0.01 & -0.01 & 0.00 & 0.01 & 0.007 & 0.077 \\
{$[\mathrm{Ba} / \mathrm{Fe}]$} & 0.13 & 0.13 & 0.10 & 0.10 & 0.08 & 0.05 & 0.18 & 0.14 & 0.08 & 0.13 & 0.11 & 0.112 & 0.035 \\
\hline
\end{tabular}


Table 7. Errors on Fe I Determination due to Parameter Uncertainties

\begin{tabular}{lccccc} 
Star & $\Delta \mathrm{T}_{\text {eff }}$ & $\Delta \log \mathrm{g}$ & $\Delta[\mathrm{Fe} / \mathrm{H}]$ & $\Delta \xi$ \\
$\pm 75 \mathrm{~K}$ & \pm 0.20 & \pm 0.10 & Total \\
& & \pm 0.20 & \\
\hline KW 23 & \pm 0.05 & $\mp 0.01$ & \pm 0.01 & \pm 0.03 & 0.060 \\
KW 30 & \pm 0.04 & $\mp 0.02$ & 0.00 & \pm 0.04 & 0.060 \\
KW 58 & \pm 0.05 & $\mp 0.01$ & \pm 0.01 & \pm 0.04 & 0.060 \\
KW 181 & \pm 0.05 & $\mp 0.01$ & 0.00 & \pm 0.01 & 0.059 \\
KW 208 & \pm 0.05 & $\mp 0.01$ & 0.00 & \pm 0.03 & 0.059 \\
KW 288 & \pm 0.04 & $\mp 0.01$ & 0.00 & \pm 0.05 & 0.059 \\
KW 301 & \pm 0.05 & $\mp 0.01$ & 0.00 & \pm 0.04 & 0.065 \\
KW 335 & \pm 0.05 & $\mp 0.01$ & 0.00 & \pm 0.03 & 0.059 \\
KW 399 & \pm 0.05 & $\mp 0.01$ & \pm 0.01 & \pm 0.03 & 0.060 \\
KW 432 & \pm 0.05 & $\mp 0.01$ & 0.00 & \pm 0.04 & 0.065 \\
KW 508 & \pm 0.05 & $\mp 0.01$ & 0.00 & \pm 0.01 & 0.060 \\
\hline
\end{tabular}


Table 8. Errors due to Uncertainties in the Stellar Parameters

\begin{tabular}{clcrrrr}
\multirow{2}{*}{ Star } & Element & $\Delta \mathrm{T}_{\text {eff }}$ & $\Delta \log \mathrm{g}$ & $\Delta[\mathrm{Fe} / \mathrm{H}]$ & \multicolumn{1}{c}{$\Delta \xi$} & Total \\
& & $\pm 75 \mathrm{~K}$ & \pm 0.20 & \pm 0.10 & \pm 0.20 & \\
\hline \multirow{2}{*}{ KW 23 } & C I & $\mp 0.06$ & \pm 0.07 & $\mp 0.01$ & 0.00 & 0.093 \\
& O I & $\mp 0.08$ & \pm 0.05 & 0.00 & $\mp 0.01$ & 0.095 \\
Na I & \pm 0.07 & 0.00 & \pm 0.03 & \pm 0.02 & 0.079 \\
Mg I & \pm 0.03 & $\mp 0.02$ & 0.00 & \pm 0.01 & 0.037 \\
Al I & \pm 0.04 & $\mp 0.01$ & 0.00 & $\mp 0.01$ & 0.042 \\
Si I & \pm 0.01 & 0.00 & \pm 0.02 & $\mp 0.01$ & 0.024 \\
Ca I & \pm 0.06 & $\mp 0.04$ & \pm 0.01 & $\mp 0.04$ & 0.083 \\
Sc II & 0.00 & \pm 0.09 & \pm 0.03 & $\mp 0.02$ & 0.097 \\
Ti I & \pm 0.07 & $\mp 0.01$ & 0.00 & $\mp 0.01$ & 0.071 \\
V I & \pm 0.09 & 0.00 & 0.00 & $\mp 0.01$ & 0.091 \\
Cr I & \pm 0.06 & $\mp 0.01$ & \pm 0.01 & $\mp 0.02$ & 0.065 \\
Ni I & \pm 0.04 & $\mp 0.01$ & \pm 0.01 & $\mp 0.05$ & 0.066 \\
Y I & \pm 0.11 & $\mp 0.01$ & 0.00 & 0.00 & 0.110 \\
Y II & $\mp 0.01$ & \pm 0.09 & $\mp 0.02$ & $\mp 0.02$ & 0.095 \\
Ba II & \pm 0.03 & \pm 0.05 & $\mp 0.11$ & $\mp 0.11$ & 0.166 \\
\hline C I & $\mp 0.04$ & \pm 0.05 & $\mp 0.01$ & $\mp 0.01$ & 0.066 \\
O I & $\mp 0.05$ & \pm 0.03 & 0.00 & $\mp 0.02$ & 0.062 \\
Na I & \pm 0.06 & 0.00 & \pm 0.02 & \pm 0.01 & 0.064 \\
Mg I & \pm 0.03 & $\mp 0.01$ & \pm 0.01 & 0.00 & 0.037 \\
Al I & \pm 0.04 & 0.00 & 0.00 & 0.00 & 0.040 \\
Si I & \pm 0.02 & $\mp 0.01$ & \pm 0.01 & $\mp 0.01$ & 0.026 \\
Ca I & \pm 0.06 & $\mp 0.04$ & 0.00 & $\mp 0.04$ & 0.082 \\
Sc II & \pm 0.01 & \pm 0.07 & \pm 0.03 & $\mp 0.02$ & 0.079 \\
Ti I & \pm 0.07 & 0.00 & 0.00 & $\mp 0.01$ & 0.071 \\
V I & \pm 0.08 & 0.00 & 0.00 & $\mp 0.01$ & 0.081 \\
Cr I & \pm 0.04 & $\mp 0.02$ & $\mp 0.01$ & $\mp 0.02$ & 0.050 \\
Ni I & \pm 0.05 & $\mp 0.01$ & 0.00 & $\mp 0.04$ & 0.065 \\
Y I & \pm 0.10 & 0.00 & 0.00 & 0.00 & 0.100 \\
Y II & 0.00 & \pm 0.08 & \pm 0.03 & $\mp 0.01$ & 0.086 \\
Ba II & \pm 0.03 & \pm 0.06 & \pm 0.04 & $\mp 0.09$ & 0.119
\end{tabular}


Table 8 - Continued

\begin{tabular}{|c|c|c|c|c|c|c|}
\hline Star & Element & $\begin{array}{c}\Delta \mathrm{T}_{\mathrm{eff}} \\
\pm 75 \mathrm{~K}\end{array}$ & $\begin{array}{l}\Delta \log \mathrm{g} \\
\pm 0.20\end{array}$ & $\begin{array}{c}\Delta[\mathrm{Fe} / \mathrm{H}] \\
\pm 0.10\end{array}$ & $\begin{array}{c}\Delta \xi \\
\pm 0.20\end{array}$ & Total \\
\hline \multirow[t]{15}{*}{ KW 335} & $\mathrm{C} \mathrm{I}$ & $\mp 0.06$ & \pm 0.06 & $\mp 0.01$ & $\mp 0.01$ & 0.086 \\
\hline & $\mathrm{O} \mathrm{I}$ & $\mp 0.06$ & \pm 0.04 & \pm 0.01 & $\mp 0.01$ & 0.074 \\
\hline & $\mathrm{Na} \mathrm{I}$ & \pm 0.07 & \pm 0.01 & \pm 0.02 & \pm 0.03 & 0.079 \\
\hline & Mg I & \pm 0.03 & $\mp 0.02$ & 0.00 & 0.00 & 0.036 \\
\hline & Al I & \pm 0.03 & $\mp 0.01$ & 0.00 & $\mp 0.01$ & 0.033 \\
\hline & Si I & \pm 0.02 & $\mp 0.01$ & \pm 0.02 & $\mp 0.01$ & 0.032 \\
\hline & $\mathrm{Ca} \mathrm{I}$ & \pm 0.05 & $\mp 0.04$ & 0.00 & $\mp 0.04$ & 0.076 \\
\hline & Sc II & 0.00 & \pm 0.09 & \pm 0.04 & $\mp 0.02$ & 0.100 \\
\hline & Ti I & \pm 0.07 & 0.00 & 0.00 & $\mp 0.01$ & 0.071 \\
\hline & V I & \pm 0.07 & $\mp 0.01$ & 0.00 & $\mp 0.02$ & 0.074 \\
\hline & Cr I & \pm 0.06 & $\mp 0.01$ & 0.00 & $\mp 0.02$ & 0.064 \\
\hline & $\mathrm{Ni} \mathrm{I}$ & \pm 0.05 & $\mp 0.01$ & \pm 0.01 & $\mp 0.04$ & 0.066 \\
\hline & Y I & \pm 0.10 & 0.00 & 0.00 & 0.00 & 0.100 \\
\hline & Y II & 0.00 & \pm 0.09 & \pm 0.03 & $\mp 0.01$ & 0.095 \\
\hline & $\mathrm{Ba}$ II & \pm 0.02 & \pm 0.05 & \pm 0.03 & $\mp 0.11$ & 0.126 \\
\hline
\end{tabular}


Table 9. Comparison of Abundances for Dwarfs and Giants

\begin{tabular}{lrrrr}
\hline \hline element & Dwarfs & $\sigma$ & Giants & $\sigma$ \\
\hline$[\mathrm{Fe} / \mathrm{H}]$ & +0.12 & 0.04 & +0.16 & 0.05 \\
{$[\mathrm{O} / \mathrm{Fe}]$} & -0.06 & 0.09 & -0.11 & 0.03 \\
{$[\mathrm{Na} / \mathrm{Fe}]$} & -0.01 & 0.03 & +0.25 & 0.06 \\
{$[\mathrm{Mg} / \mathrm{Fe}]$} & 0.00 & 0.04 & +0.27 & 0.05 \\
{$[\mathrm{Al} / \mathrm{Fe}]$} & 0.00 & 0.04 & 0.00 & 0.03 \\
{$[\mathrm{Si} / \mathrm{Fe}]$} & -0.01 & 0.03 & +0.06 & 0.02 \\
{$[\mathrm{Ca} / \mathrm{Fe}]$} & -0.01 & 0.05 & -0.08 & 0.02 \\
{$[\mathrm{Sc} / \mathrm{Fe}]$} & +0.04 & 0.02 & -0.04 & 0.05 \\
{$[\mathrm{Ti} / \mathrm{Fe}]$} & -0.04 & 0.06 & -0.07 & 0.03 \\
{$[\mathrm{~V} / \mathrm{Fe}]$} & +0.04 & 0.04 & +0.06 & 0.03 \\
{$[\mathrm{Cr} / \mathrm{Fe}]$} & +0.00 & 0.03 & +0.05 & 0.01 \\
{$[\mathrm{Ni} / \mathrm{Fe}]$} & -0.03 & 0.03 & +0.02 & 0.02 \\
{$[\mathrm{Y} / \mathrm{Fe}]$} & +0.01 & 0.08 & -0.11 & 0.01 \\
{$[\mathrm{Ba} / \mathrm{Fe}]$} & +0.11 & 0.04 & +0.33 & 0.05 \\
\hline
\end{tabular}




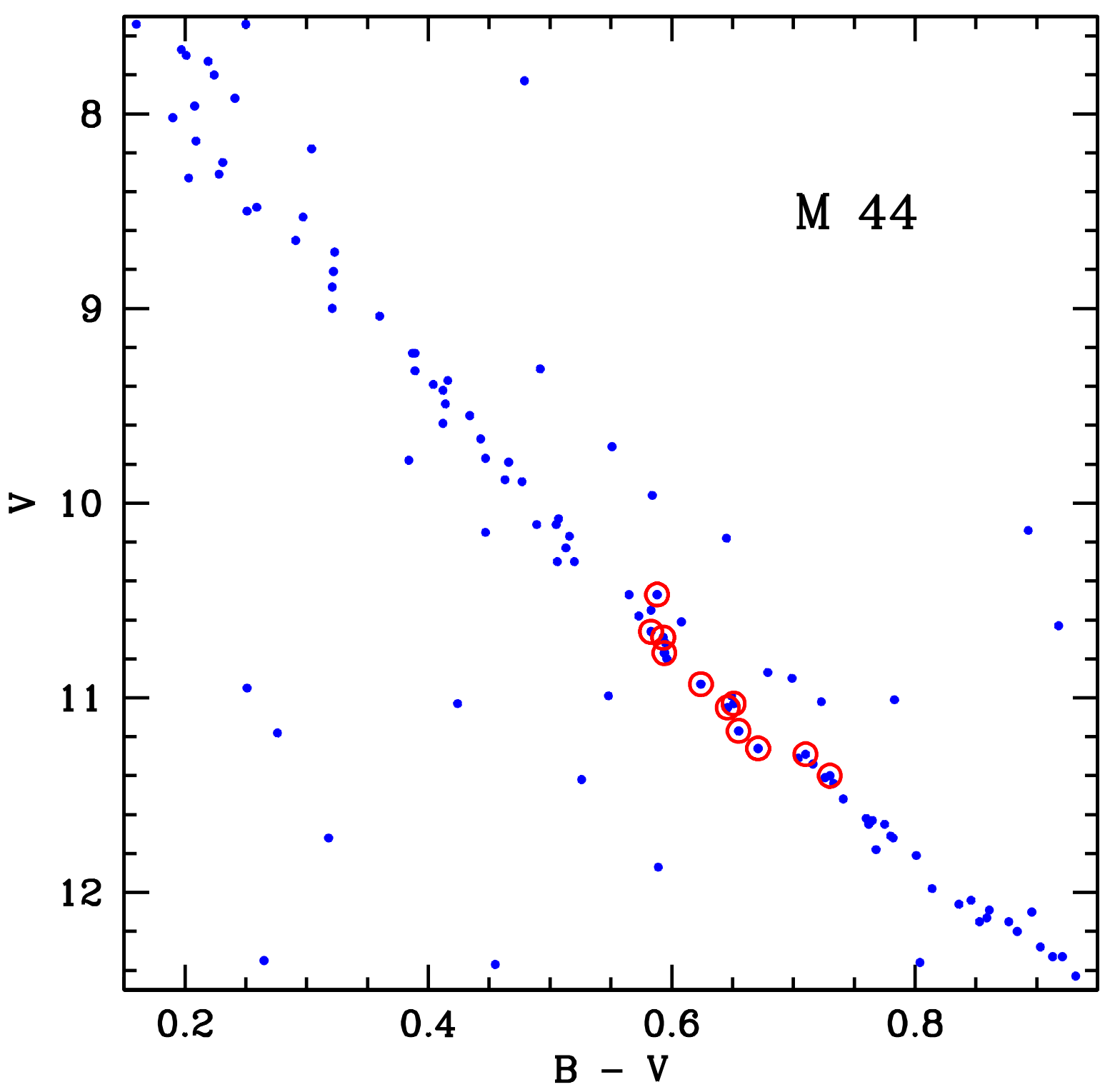

Fig. 1.- The color-magnitude diagram for Praesepe. The data are from Johnson (1952) and Mendoza (1967). The stars we have observed are circled. 


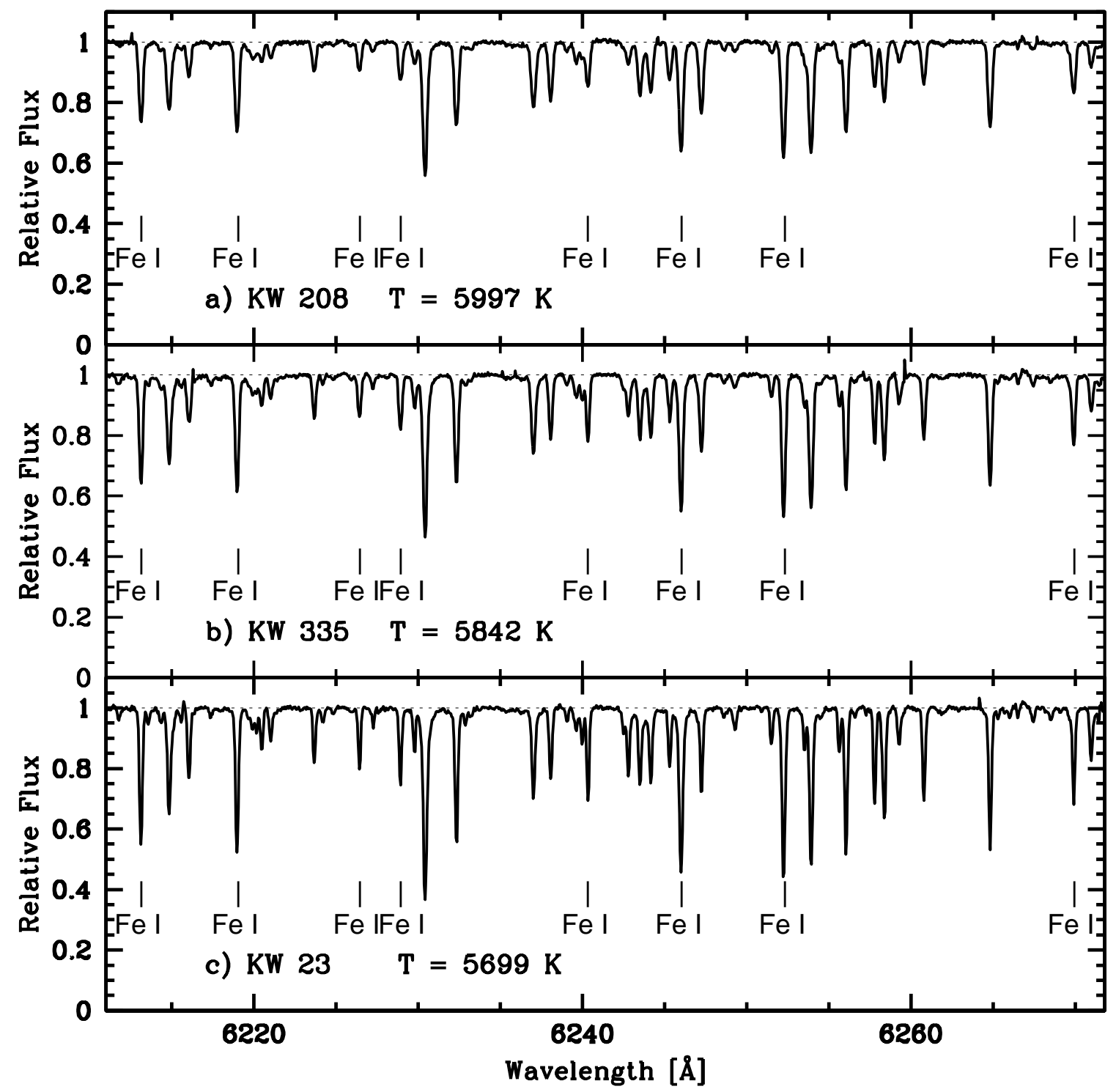

Fig. 2.- Samples of our spectra in the $6200 \AA$ region. The three stars cover a range in temperature and the $\mathrm{Fe} I$ lines indicated clearly become stronger as the temperature decreases. The $\mathrm{S} / \mathrm{N}$ per pixel for these spectra is $\sim 150$. The continuum is shown as a light dotted line at 1.0. 


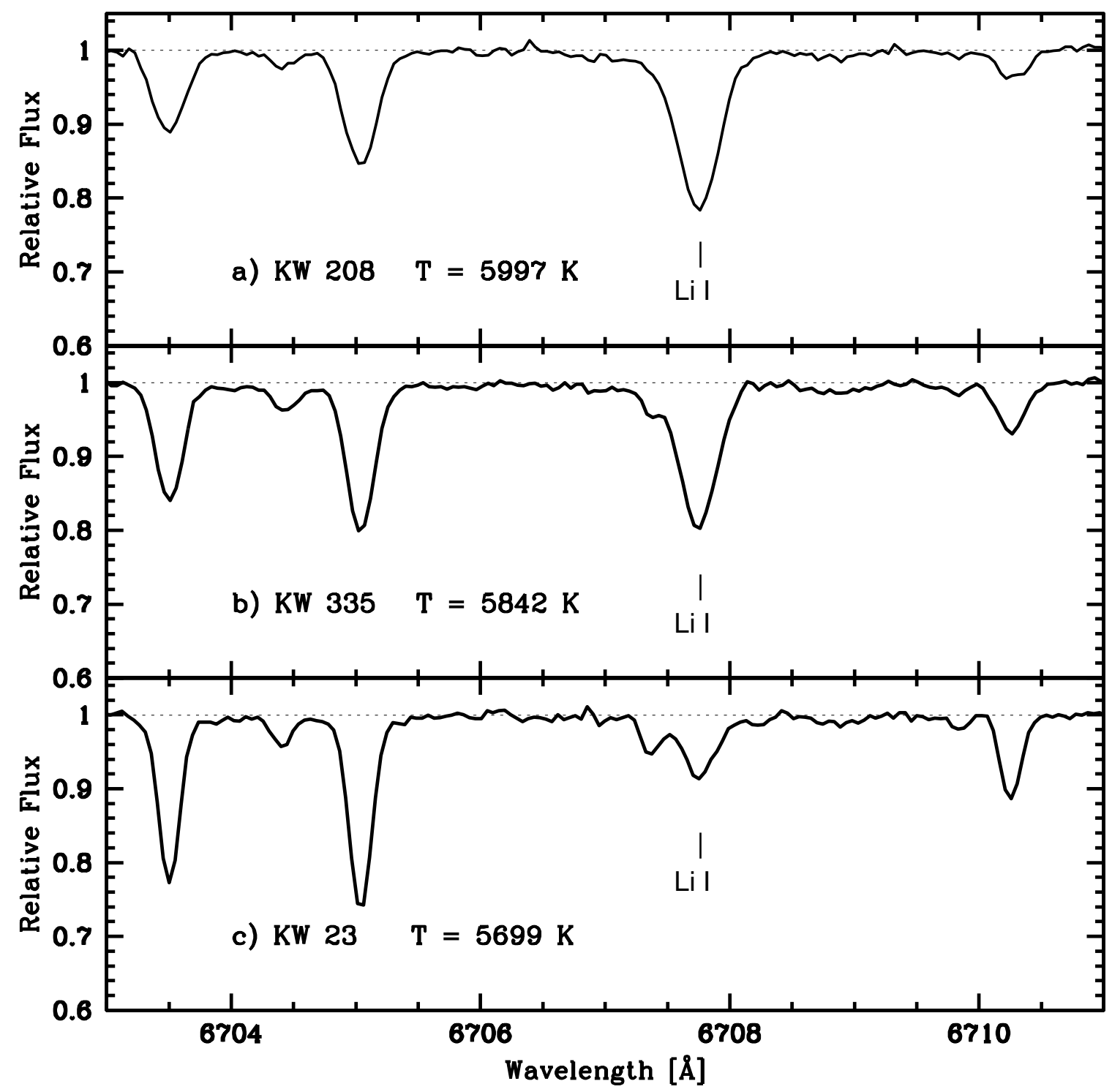

Fig. 3.- Samples of our spectra in an $8 \AA$ region around the Li I doublet. With decreasing temperature the $\mathrm{Li}$ I feature decreases due to Li depletion while the blending line increases in strength. The lines at 6703,6705 , and $6710 \AA$ are due to Fe I. The continuum is shown as a light dotted line at 1.0. The vertical scale in this figure covers a smaller range than the one in Figure 2. 


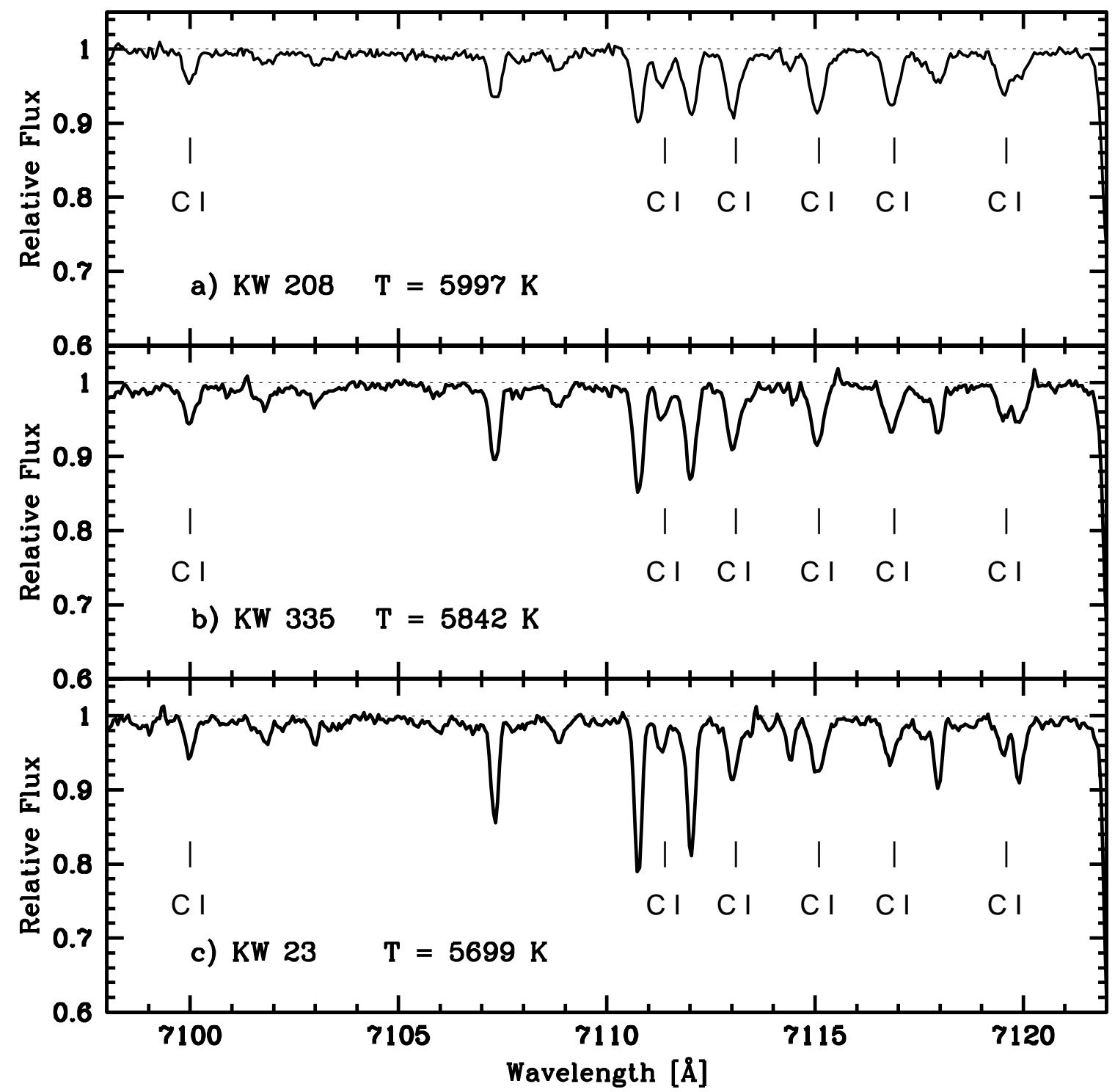

Fig. 4.- Samples of our spectra in a $24 \AA$ region which contains several high excitation C I lines. Although the lines weaken with decreasing temperature, they are not very sensitive to temperature. The continuum is shown as a light dotted line at 1.0. 


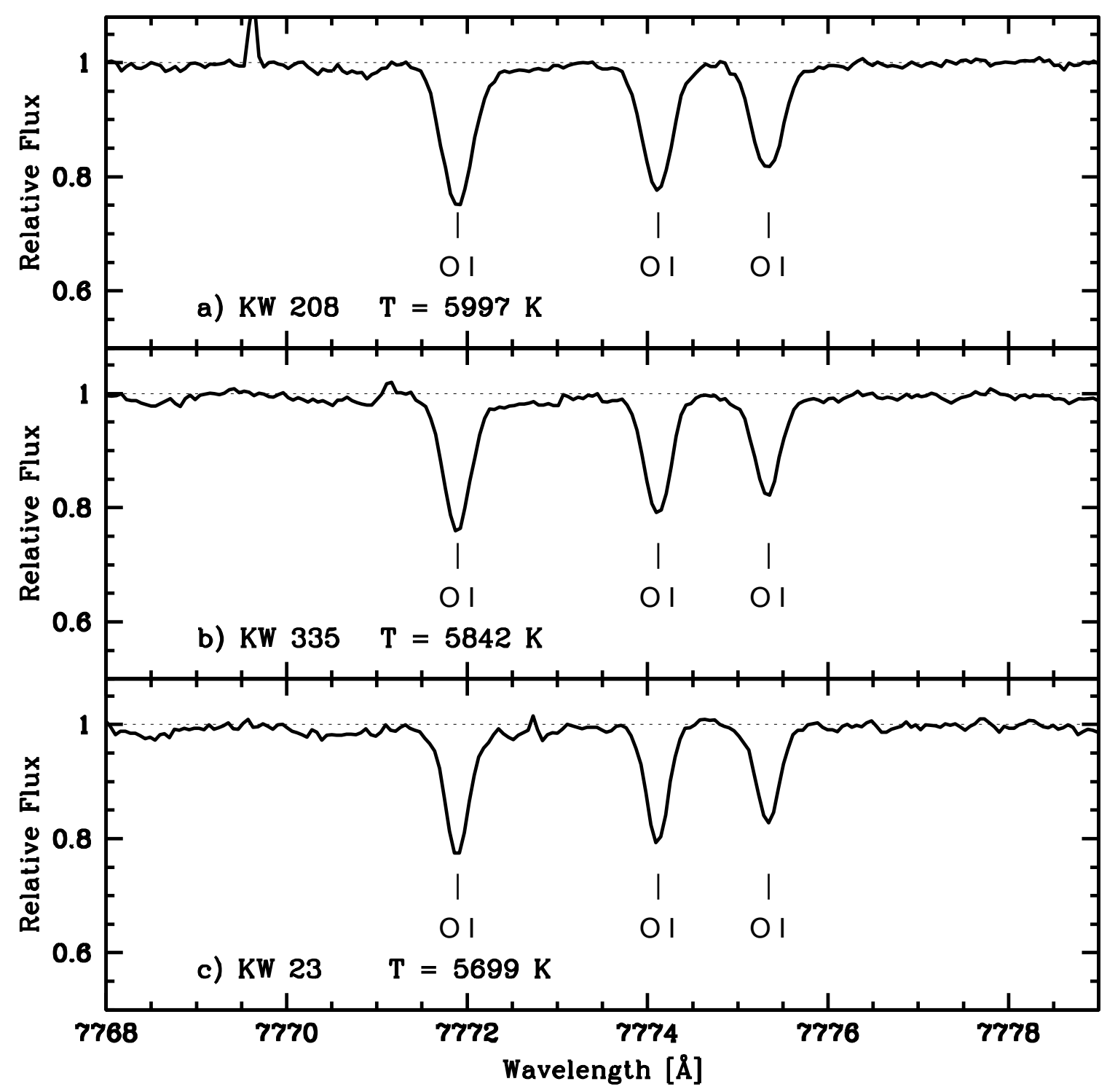

Fig. 5.- Samples of our spectra in an $11 \AA$ region surrounding the O I triplet. The continuum is shown as a light dotted line at 1.0. 


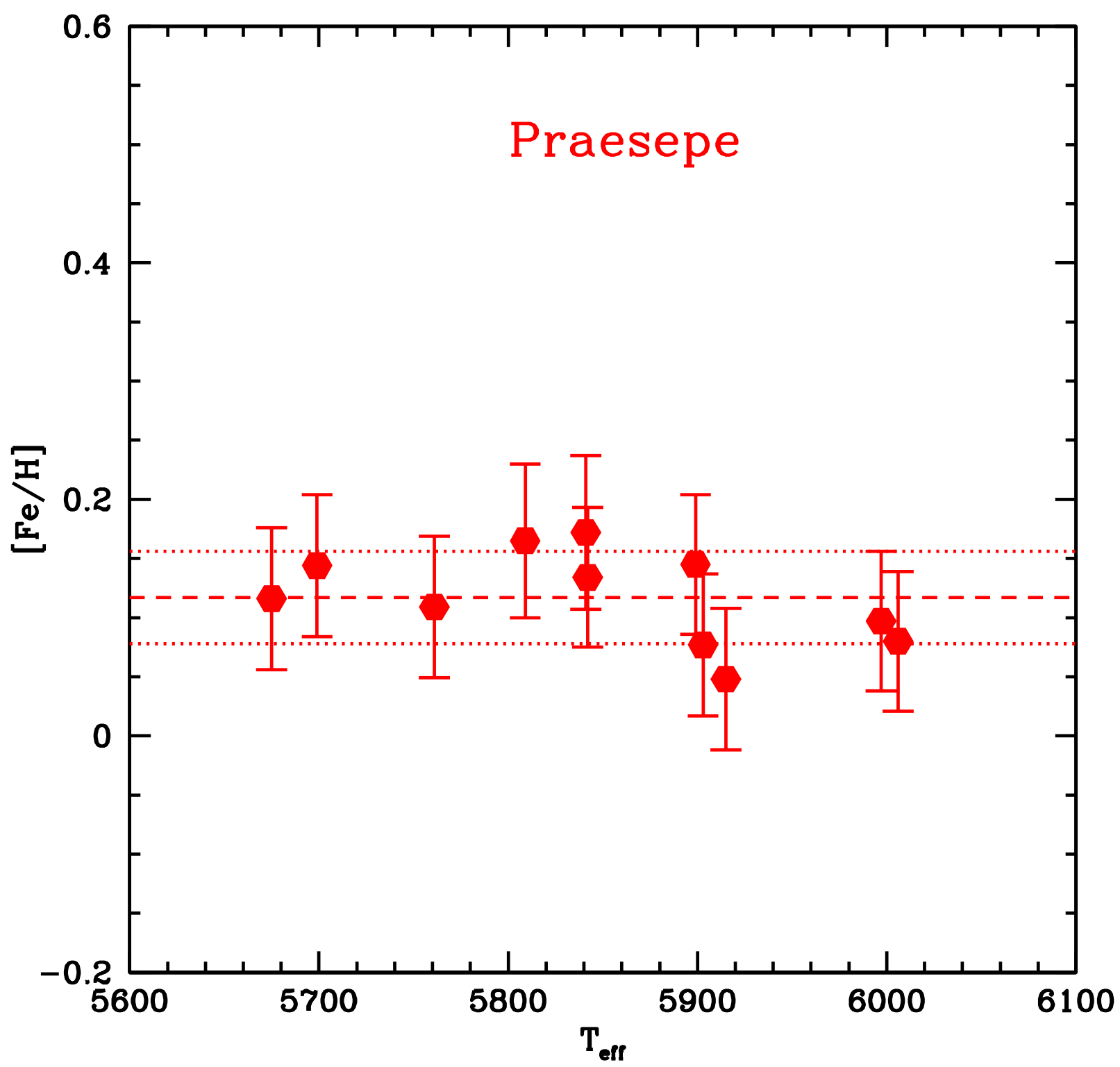

Fig. 6.- The abundance of Fe in our Praesepe stars. The error bars are those due to the uncertainties in the stellar parameters. The dotted line shows the mean $[\mathrm{Fe} / \mathrm{H}]=0.117$ \pm 0.039 . The dashed lines are that $1 \sigma$ error. 


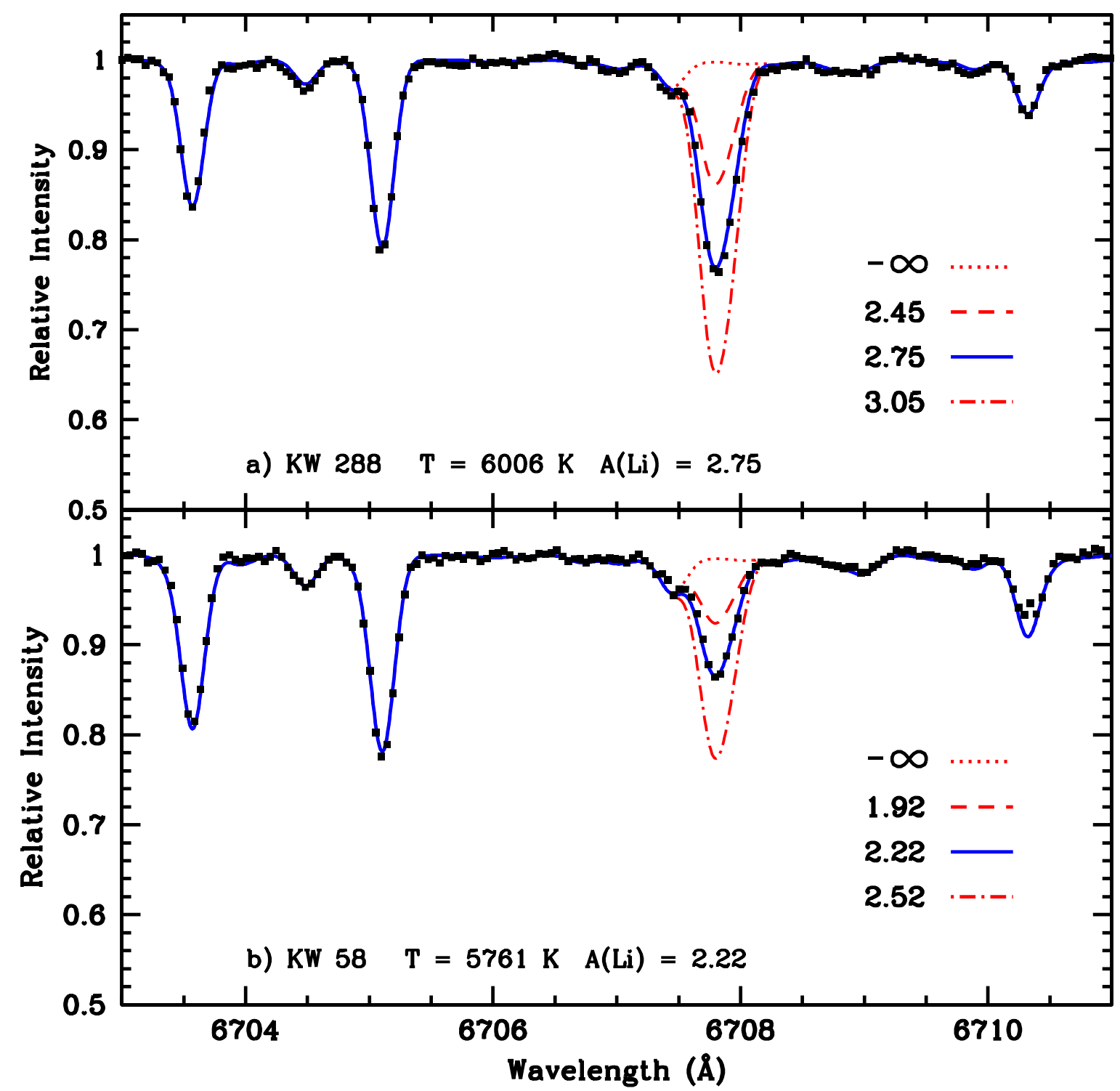

Fig. 7.- Examples of the synthesized spectra in the Li regions for two Praesepe stars. The (black) squares are the observed spectra, the solid (blue) line is the best synthetic fit, the dot-dash (red) line is a factor of two more Li, the dashed (red) line is a factor of two less Li and the dotted (red) shows the spectrum with no Li. The other lines in this spectra region are well matched by the synthesis. 


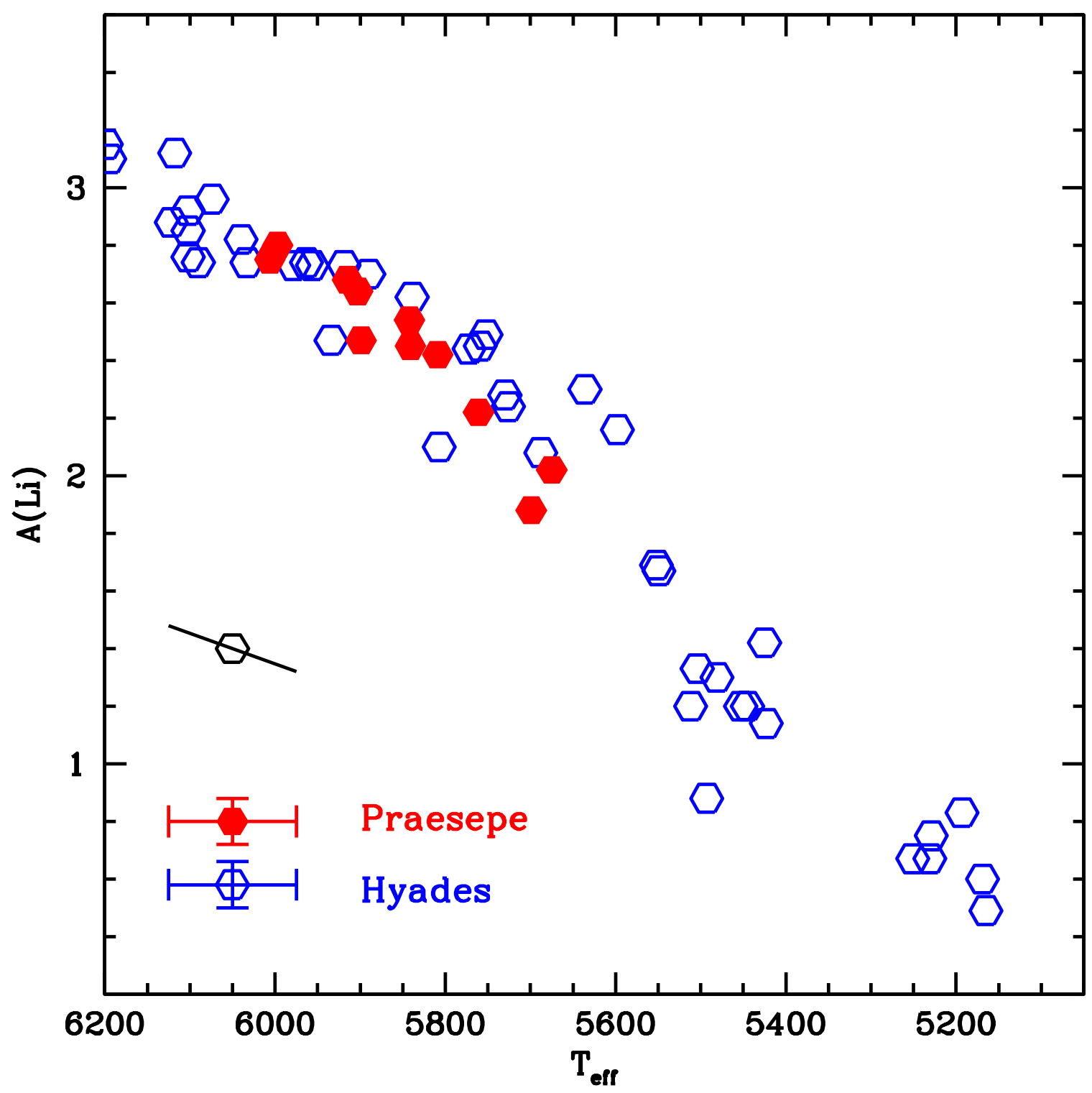

Fig. 8.- Abundances of Li in the Hyades and Praesepe. The Praesepe stars are shown as filled hexagons while the Hyades stars are open hexagons. A typical uncertainty in both $\mathrm{A}(\mathrm{Li})$ and $T_{\text {eff }}$ is shown in the lower left. The symbol above those error bars shows the direction and change in $\mathrm{A}(\mathrm{Li})$ due to an uncertainty in $\mathrm{T}_{\text {eff }}$ of $\pm 75 \mathrm{~K}$. 


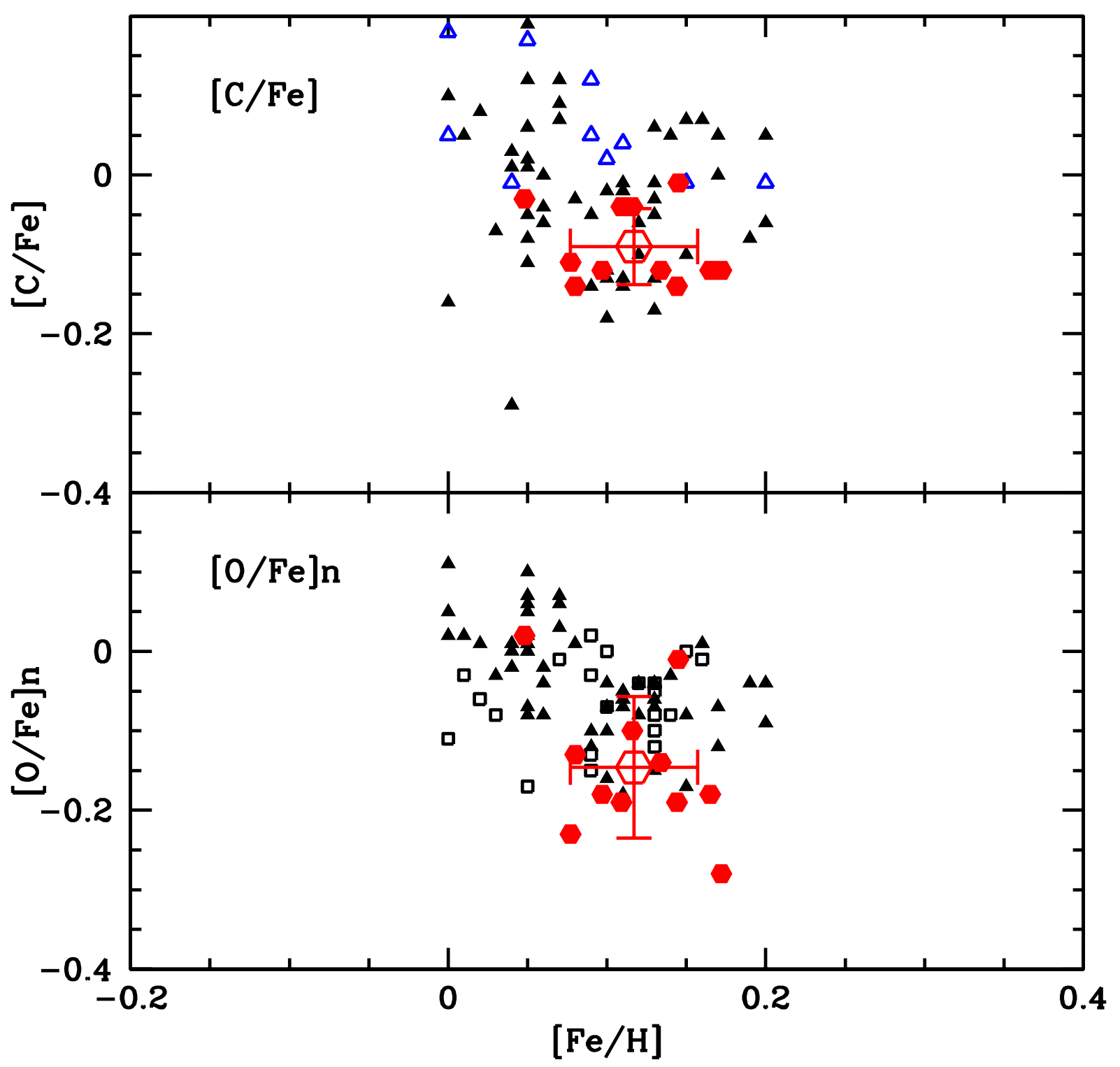

Fig. 9.- Abundance ratios for $\mathrm{C}$ and $\mathrm{O}$. The Praesepe stars are the solid hexagons (red) and the large open hexagon is the mean value for $[\mathrm{X} / \mathrm{Fe}]$ with the errorbars showing the standard deviation in the mean of the two coordinates. The Edvardsson et al. (1993) abundances are the open squares, the Reddy et al. $(2003,2006)$ are the open triangles (blue), while the Takeda \& Honda (2005) are filled triangles. 


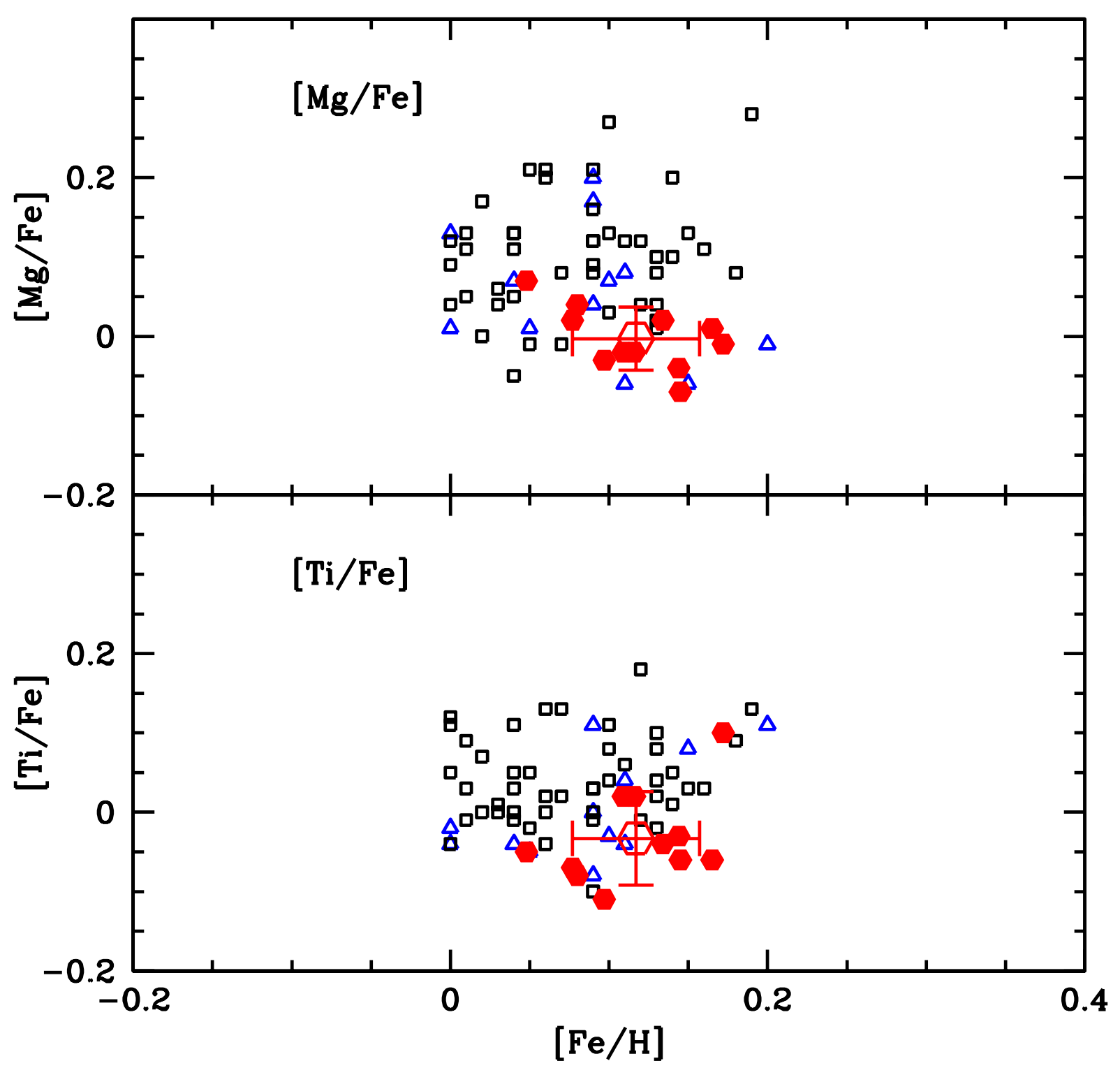

Fig. 10.- Abundances of the alpha-elements, $\mathrm{Mg}$ and Ti, relative to Fe. The filled hexagons are the Praesepe stars and the large open hexagon is the mean value for $[\mathrm{X} / \mathrm{Fe}]$ with the errorbars showing the standard deviation in the mean of the two coordinates. The open squares are the selected sample of field stars from Edvardsson et al. (1993) and the open triangles (blue) are from the two Reddy et al. papers. 


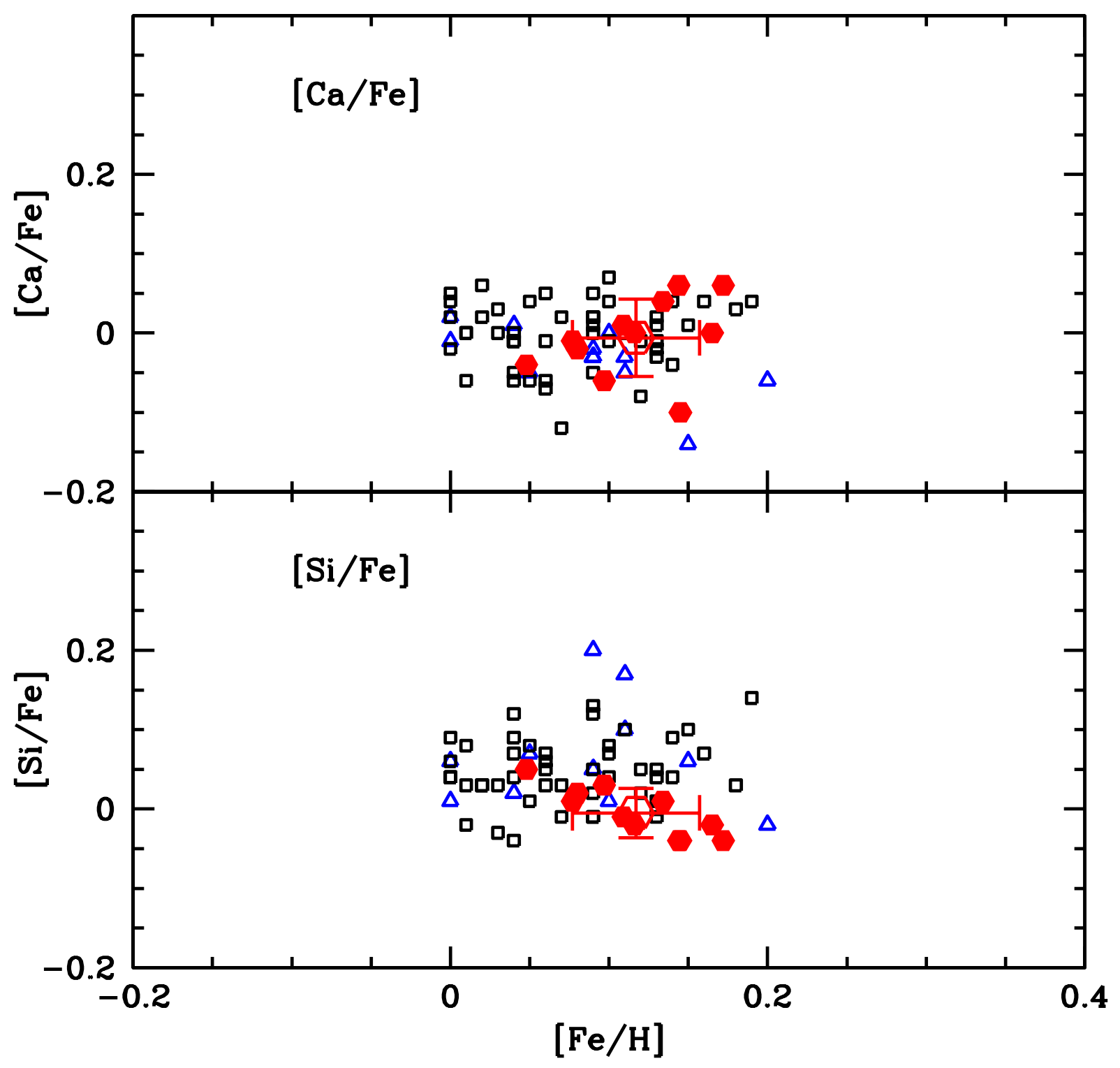

Fig. 11.- Abundances of the alpha-elements, $\mathrm{Ca}$ and Si, relative to Fe. The Praesepe stars are the solid hexagons, the Edvardsson et al. (1993) are the open squares, the Reddy et al. $(2003,2006)$ are the open triangles (blue). 


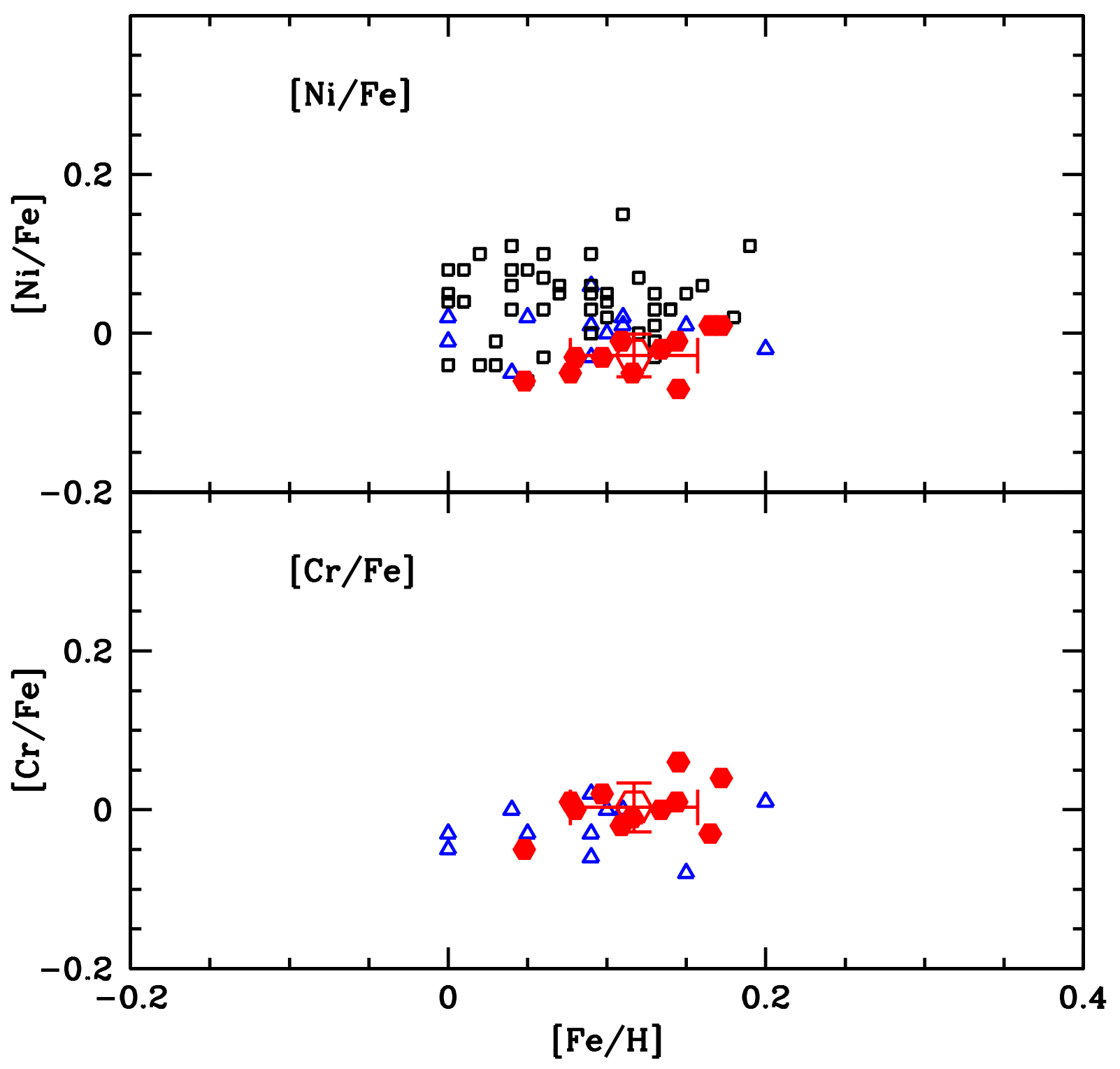

Fig. 12.- Abundances of two Fe-peak elements, Cr and Ni relative to Fe. The Praesepe stars are the solid hexagons, the Edvardsson et al. (1993) are the open squares for $\mathrm{Ni}$; they did not measure Cr. The open triangles (blue) are from the two Reddy et al. papers. 


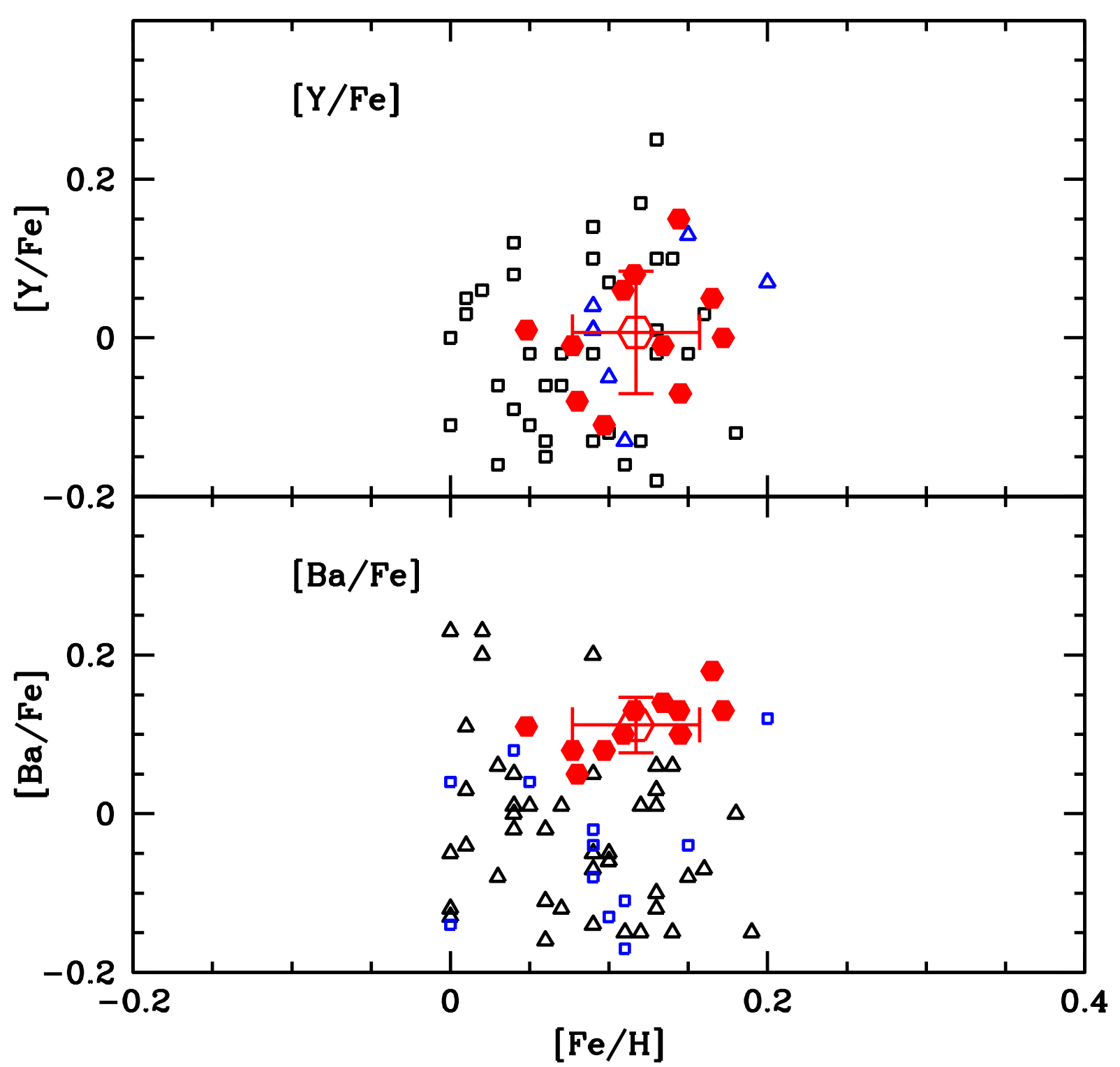

Fig. 13.- Abundances of $\mathrm{Y}$ and Ba relative to Fe. Praesepe stars are the solid hexagons; the Edvardsson et al. (1993) are the open squares; the open triangles (blue) are from the two Reddy et al. papers. Our Y results are in accord with the field star results. Our values for $[\mathrm{Ba} / \mathrm{Fe}]$ at $+0.11 \pm 0.04$ are in the upper range of the field star values. 


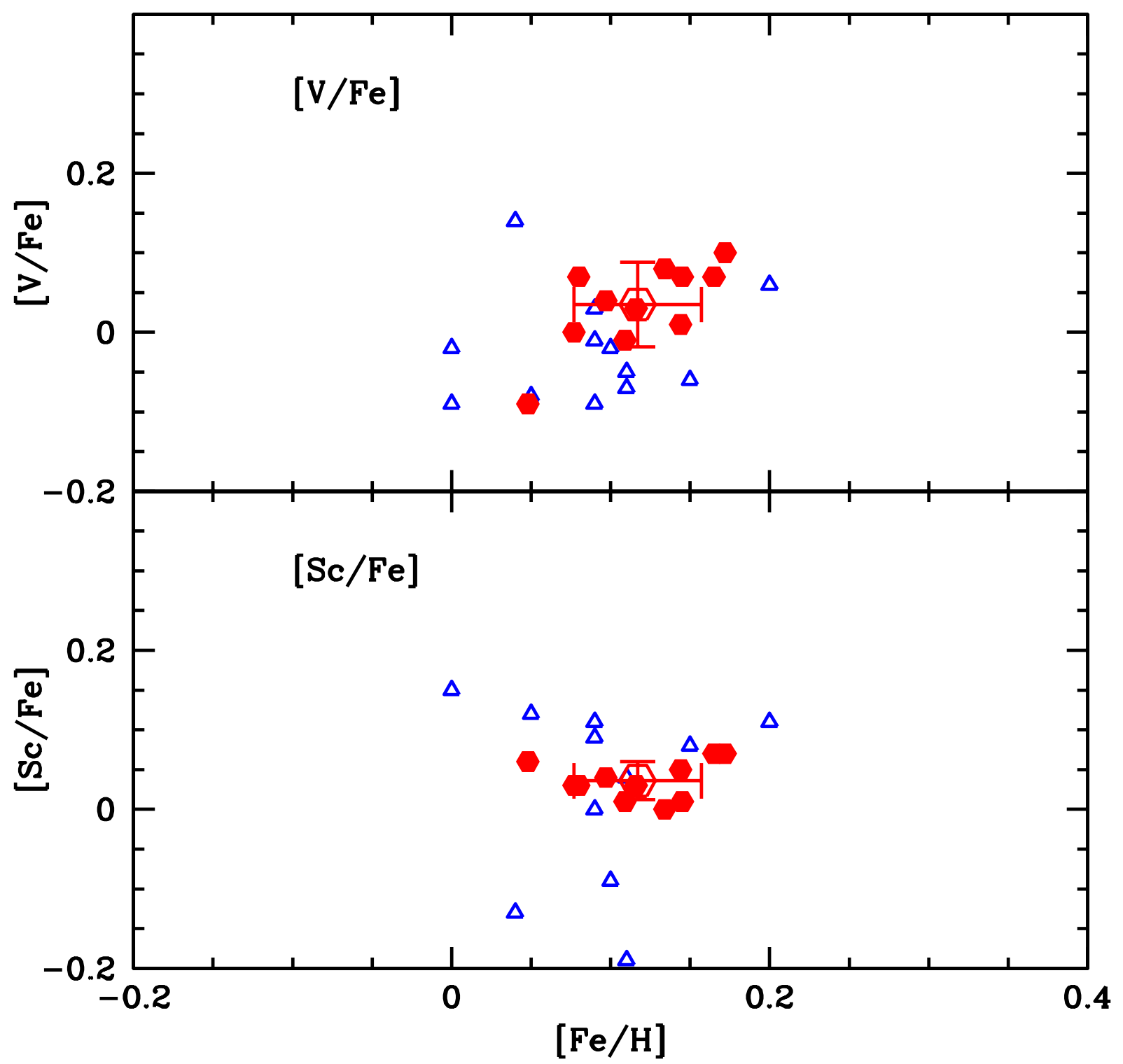

Fig. 14.- Abundances of $\mathrm{V}$ and Sc relative to Fe. The solid hexagons are the Praesepe stars; open triangles are the field stars are from Reddy et al. (2003, 2006), Both samples are solar within the errors. 


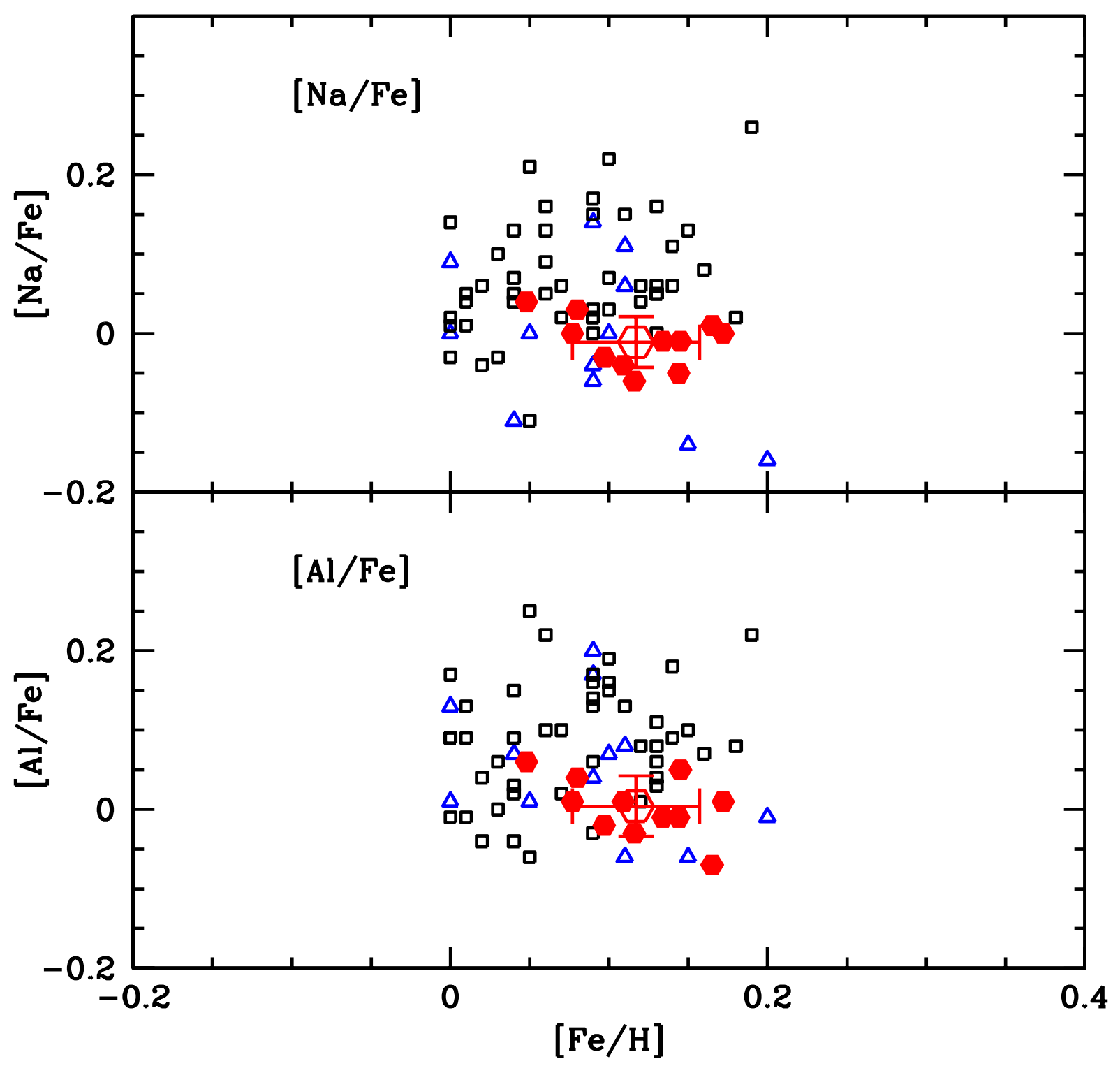

Fig. 15.- Abundances of $\mathrm{Al}$ and Na relative to Fe. The solid hexagons are the Praesepe stars; the open triangles are the field stars are from Reddy et al. $(2003,2006)$, open squares are from Edvardsson et al. (1993). Both $\mathrm{Al}$ and $\mathrm{Na}$ are solar which is generally lower than the filed stars. 


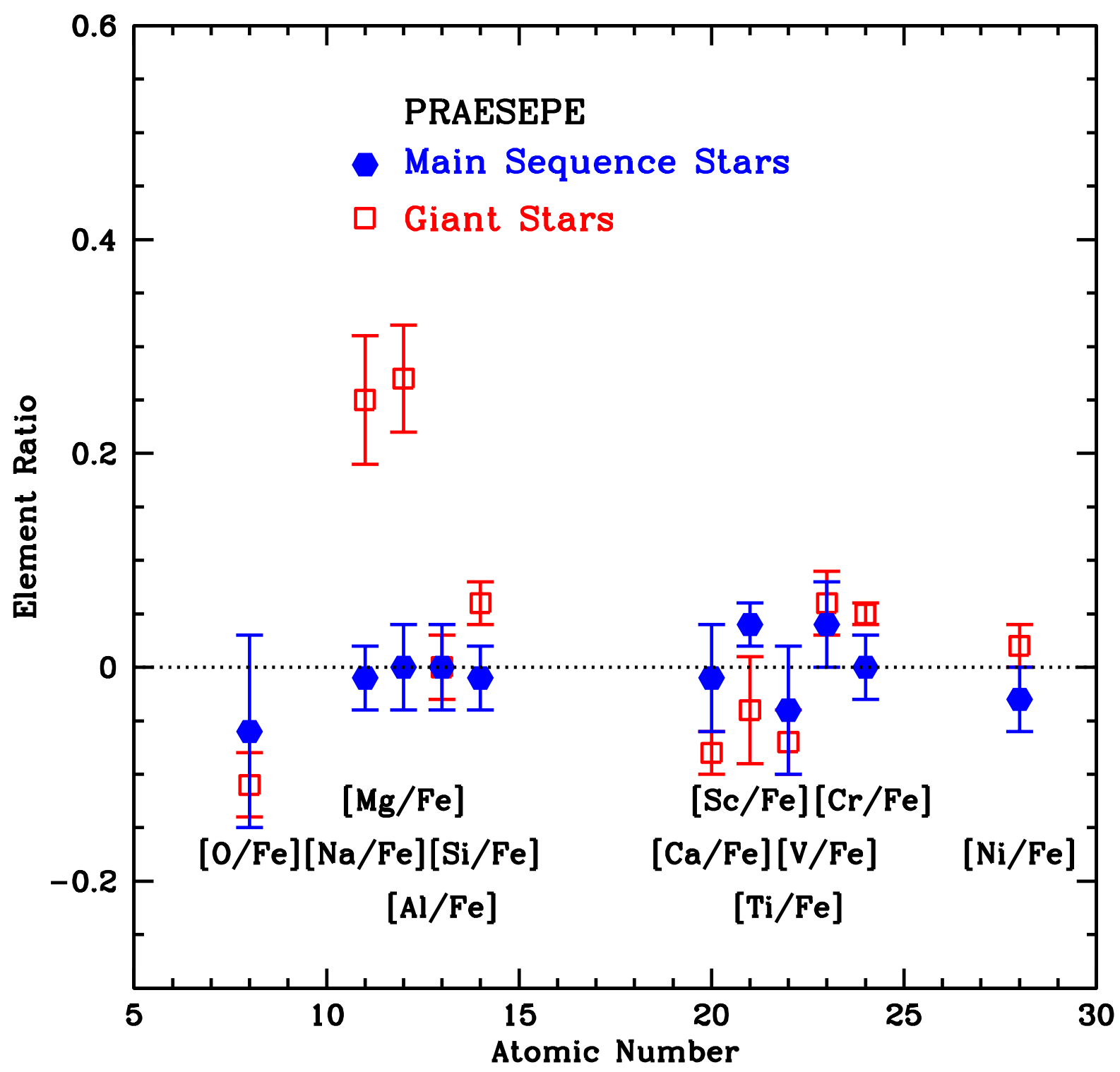

Fig. 16.- Abundance averages in our 11 main sequence stars compared with the abundance average in the three red giants of Carrera \& Pancino (2011). Our dwarfs with 1 sigma error bars are the (blue) hexagons while the results for the giants are shown by the (red) open squares. The horizontal line represents the solar values. With the exception of $\mathrm{Na}$ and $\mathrm{Mg}$ the agreement is very good. See text for discussion. 
Table APPENDIX. Spectral Lines Used and Measured Equivalent Widths in Three Stars

\begin{tabular}{ccccccc}
\hline \hline \multirow{2}{*}{ Ion } & $\lambda(\AA)$ & Ex. Pot. $(\mathrm{eV})$ & $\log g f$ & $\mathrm{KW} 23$ & $\mathrm{KW} 208$ & $\mathrm{KW} 335$ \\
\hline \multirow{2}{*}{ C I } & 6587.62 & 8.53 & -1.000 & 13.1 & 19.6 & $\ldots$ \\
& 7100.13 & 8.64 & -1.020 & 11.8 & $\ldots$ & 17.8 \\
& 7111.45 & 8.64 & -1.080 & 10.8 & 16.6 & 13.5 \\
& 7113.17 & 8.65 & -0.770 & 24.0 & 29.1 & 28.6 \\
& 7115.17 & 8.64 & -0.930 & 25.3 & 32.6 & $\ldots$ \\
& 7116.96 & 8.65 & -0.900 & 18.0 & 27.5 & 19.9 \\
& 7119.70 & 8.64 & -1.220 & 11.3 & $\ldots$ & $\ldots$ \\
\hline O I & 6158.17 & 10.74 & -0.320 & $\ldots$ & 5.7 & $\ldots$ \\
& 7771.94 & 9.11 & +0.369 & 72.8 & 107.2 & 90.5 \\
& 7774.17 & 9.11 & +0.223 & 60.2 & 91.7 & 75.1 \\
& 7775.39 & 9.11 & +0.002 & 53.3 & 78.5 & 61.6 \\
\hline Na I & 6154.227 & 2.10 & -1.660 & 46.6 & 35.5 & 41.6 \\
& 6160.751 & 2.10 & -1.350 & 65.8 & 51.8 & 64.4 \\
\hline Mg I & 6965.41 & 5.75 & -1.870 & 27.6 & 21.0 & 26.5 \\
& 7387.70 & 5.75 & -1.200 & 87.4 & 74.8 & 90.2 \\
\hline Al I & 6698.67 & 3.14 & -1.950 & 25.7 & 18.2 & 22.4 \\
\hline Si I & 5772.15 & 5.08 & -1.750 & 61.2 & 57.3 & 61.2 \\
& 5948.55 & 5.08 & -1.225 & 98.2 & 94.1 & 97.2 \\
& 6125.03 & 5.61 & -1.540 & 39.3 & 37.7 & 39.4 \\
& 6142.49 & 5.62 & -1.480 & 40.0 & 41.0 & 42.0 \\
& 6155.14 & 5.62 & -0.840 & 98.4 & 97.1 & 96.2 \\
& 6848.57 & 5.86 & -1.740 & 20.4 & 19.1 & 20.6 \\
& 7003.57 & 5.96 & -0.860 & 68.6 & 71.5 & 70.5 \\
& 7005.90 & 5.98 & -0.680 & 91.4 & 94.6 & 93.8 \\
& 7289.19 & 5.62 & -0.620 & $\ldots$ & $\ldots$ & $\ldots$ \\
& 7405.79 & 5.61 & -0.570 & 102.1 & 100.4 & 100.6 \\
& 7760.64 & 6.20 & -1.280 & 18.3 & 24.9 & 32.6 \\
\hline \multirow{6}{*}{ Ca I } & 6161.30 & 2.52 & -1.270 & 84.8 & 58.9 & 77.8 \\
& 6169.04 & 2.52 & -0.797 & $\ldots$ & 97.4 & 107.0
\end{tabular}


Table APPENDIX — Continued

\begin{tabular}{|c|c|c|c|c|c|c|}
\hline Ion & $\lambda(\AA)$ & Ex. Pot. (eV) & $\log g f$ & KW 23 & KW 208 & KW 335 \\
\hline & 6169.56 & 2.53 & -0.374 & $\cdots$ & 115.1 & $\cdots$ \\
\hline & 6449.81 & 2.52 & -0.502 & $\cdots$ & 109.9 & $\cdots$ \\
\hline & 6455.60 & 2.52 & -1.340 & 69.0 & 51.9 & 67.1 \\
\hline & 6464.68 & 2.52 & -2.530 & 22.1 & 9.4 & 15.5 \\
\hline & 6471.66 & 2.53 & -0.638 & $\ldots$ & 96.5 & 102.6 \\
\hline & 6499.65 & 2.52 & -0.818 & 103.2 & 89.6 & 96.2 \\
\hline \multirow[t]{2}{*}{$\overline{\mathrm{Sc} \text { II }}$} & 6245.61 & 1.51 & -1.150 & 37.6 & 38.6 & 36.9 \\
\hline & 6604.60 & 1.36 & -1.230 & 39.7 & 40.2 & 38.1 \\
\hline \multirow[t]{11}{*}{ Ti I } & 5866.46 & 1.07 & -0.870 & 57.7 & 38.6 & 51.0 \\
\hline & 6126.22 & 1.07 & -1.460 & 30.5 & 15.5 & 22.6 \\
\hline & 6554.24 & 1.44 & -1.160 & 23.2 & 7.8 & 13.6 \\
\hline & 6556.08 & 1.46 & -1.100 & 26.3 & 13.9 & 19.6 \\
\hline & 6599.11 & 0.90 & -2.060 & 13.5 & $\ldots$ & 9.6 \\
\hline & 6745.55 & 2.24 & -1.100 & 4.2 & $\cdots$ & $\cdots$ \\
\hline & 7138.93 & 1.44 & -1.720 & 11.0 & $\ldots$ & 7.1 \\
\hline & 7216.19 & 1.44 & -1.300 & 26.3 & 10.8 & 15.9 \\
\hline & 7251.72 & 1.43 & -0.860 & 54.7 & 35.4 & 47.7 \\
\hline & 7440.58 & 2.25 & -1.080 & 7.6 & $\ldots$ & 9.0 \\
\hline & 7949.15 & 1.50 & -1.430 & 15.8 & $\cdots$ & 10.5 \\
\hline \multirow[t]{2}{*}{ V I } & 6216.31 & 0.28 & -0.830 & 48.1 & 33.8 & 45.3 \\
\hline & 6504.14 & 1.18 & -0.740 & 18.2 & 11.1 & 15.9 \\
\hline \multirow[t]{9}{*}{ Cr I } & 5783.09 & 3.32 & -0.500 & 45.4 & 33.1 & 38.0 \\
\hline & 5783.89 & 3.32 & -0.295 & 60.8 & 43.7 & 53.8 \\
\hline & 6330.09 & 0.94 & -2.920 & 39.5 & $\ldots$ & 34.5 \\
\hline & 6501.20 & 0.98 & -3.660 & 12.6 & $\ldots$ & 12.0 \\
\hline & 6605.57 & 4.14 & -0.810 & $\cdots$ & $\ldots$ & $\ldots$ \\
\hline & 6630.03 & 1.03 & -3.560 & 12.3 & 9.3 & 10.7 \\
\hline & 6978.48 & 3.46 & +0.143 & 81.1 & 65.7 & 70.9 \\
\hline & 6979.81 & 3.46 & -0.460 & 49.2 & 38.5 & 43.3 \\
\hline & 6980.91 & 3.46 & -1.120 & 16.4 & 10.3 & 9.6 \\
\hline Fe I & 5775.08 & 4.22 & -1.300 & 70.5 & 59.5 & 65.4 \\
\hline
\end{tabular}


Table APPENDIX - Continued

\begin{tabular}{rrrrrr}
\hline \hline Ion $\lambda(\AA)$ & Ex. Pot. $(\mathrm{eV})$ & $\log g f$ & KW 23 & KW 208 & KW 335 \\
\hline 5809.22 & 3.88 & -1.760 & 64.5 & 51.6 & 59.0 \\
5849.69 & 3.69 & -2.970 & 11.2 & 5.1 & 8.8 \\
5852.22 & 4.55 & -1.220 & 51.9 & 41.4 & 47.7 \\
5855.08 & 4.61 & -1.560 & 28.5 & 22.3 & 25.5 \\
5856.09 & 4.29 & -1.600 & 42.5 & 33.4 & 39.1 \\
5858.78 & 4.22 & -2.190 & 17.6 & 14.3 & 17.6 \\
5859.59 & 4.55 & -0.610 & 83.3 & 76.2 & 82.8 \\
5861.11 & 4.28 & -2.340 & 10.4 & 7.8 & 8.9 \\
5862.36 & 4.55 & -0.420 & 101.8 & 93.3 & 102.1 \\
6027.05 & 4.08 & -1.150 & 73.8 & 66.6 & 72.8 \\
6055.99 & 4.73 & -0.460 & 84.8 & 78.9 & 82.2 \\
6127.90 & 4.14 & -1.399 & 58.7 & 50.7 & 55.2 \\
6151.62 & 2.18 & -3.299 & 59.8 & 44.1 & 55.8 \\
6157.73 & 4.07 & -1.270 & 71.9 & 65.0 & 66.7 \\
6159.38 & 4.61 & -1.880 & 16.8 & 10.9 & 13.8 \\
6165.36 & 4.14 & -1.580 & 53.3 & 42.6 & 51.1 \\
6173.34 & 2.22 & -2.880 & 81.1 & 69.5 & 75.8 \\
6180.20 & 2.73 & -2.620 & 68.7 & 58.2 & 64.9 \\
6213.44 & 2.22 & -2.660 & 98.8 & 81.0 & 96.8 \\
6219.29 & 2.20 & -2.433 & 107.4 & 93.9 & 103.2 \\
6226.74 & 3.88 & -2.220 & 37.1 & 27.2 & 36.8 \\
6229.23 & 2.84 & -3.020 & 50.5 & 37.2 & 47.6 \\
6240.65 & 2.22 & -3.200 & 59.8 & 45.6 & 55.8 \\
6270.23 & 2.86 & -2.690 & 59.8 & 54.6 & 57.4 \\
6271.28 & 3.33 & -2.840 & 35.0 & 24.6 & 31.2 \\
6290.97 & 4.73 & -0.760 & 80.8 & 67.9 & 73.3 \\
6322.68 & 2.59 & -2.450 & 88.9 & 73.3 & 81.4 \\
6330.85 & 4.73 & -1.280 & 44.4 & 37.7 & 39.0 \\
6335.34 & 2.20 & -2.230 & $\ldots$ & 98.6 & 106.9 \\
6344.15 & 2.43 & -2.900 & 68.8 & 60.7 & 69.0 \\
6380.75 & 4.19 & -1.410 & 62.2 & 49.5 & 58.9
\end{tabular}


Table APPENDIX - Continued

\begin{tabular}{|c|c|c|c|c|c|c|}
\hline Ion & $\lambda(\AA)$ & Ex. Pot. (eV) & $\log g f$ & KW 23 & KW 208 & KW 335 \\
\hline & 6392.54 & 2.28 & -4.030 & 26.2 & 13.4 & 16.9 \\
\hline & 6481.87 & 2.28 & -2.980 & 77.1 & 61.8 & 67.4 \\
\hline & 6498.94 & 0.96 & -4.690 & 56.5 & 38.3 & 49.3 \\
\hline & 6581.22 & 1.48 & -4.790 & 28.2 & 19.1 & 23.9 \\
\hline & 6591.33 & 4.59 & -2.070 & 12.1 & 7.9 & 10.8 \\
\hline & 6608.04 & 2.28 & -4.020 & 28.1 & 12.9 & 17.7 \\
\hline & 6625.04 & 1.01 & -5.350 & 27.9 & 11.0 & 17.9 \\
\hline & 6627.56 & 4.55 & -1.610 & 40.1 & 28.6 & 32.1 \\
\hline & 6703.57 & 2.76 & -3.130 & 45.6 & 34.0 & 39.0 \\
\hline & 6705.12 & 4.61 & -1.170 & 57.8 & 49.7 & 51.1 \\
\hline & 6726.67 & 4.61 & -1.160 & 57.0 & 49.2 & 55.5 \\
\hline & 6750.15 & 2.42 & -2.620 & 85.8 & 70.1 & 80.4 \\
\hline & 7071.87 & 4.61 & -1.700 & 32.5 & 29.6 & 32.4 \\
\hline & 7107.47 & 4.19 & -2.070 & 30.1 & 20.1 & 27.4 \\
\hline & 7127.57 & 4.99 & -1.250 & 36.0 & 28.4 & 34.7 \\
\hline & 7130.93 & 4.22 & -0.740 & 108.1 & 93.5 & 99.8 \\
\hline & 7132.98 & 4.07 & -1.770 & 51.8 & 44.3 & 50.4 \\
\hline & 7142.52 & 4.95 & -1.090 & 47.6 & 38.9 & 44.3 \\
\hline & 7155.63 & 5.01 & -1.090 & 48.1 & 36.9 & 43.6 \\
\hline & 7745.52 & 5.08 & -1.180 & 31.0 & 32.3 & 34.9 \\
\hline & 7746.60 & 5.06 & -1.290 & 28.8 & 28.3 & 26.7 \\
\hline & 7751.11 & 4.99 & -0.770 & 58.2 & 57.8 & 57.5 \\
\hline & 7879.78 & 5.03 & -1.650 & 15.3 & 9.3 & 11.3 \\
\hline \multirow[t]{6}{*}{ Fe II } & 6149.25 & 3.89 & -2.724 & 39.6 & 48.3 & 43.5 \\
\hline & 6247.56 & 3.89 & -2.329 & 58.4 & 71.9 & 64.9 \\
\hline & 6456.39 & 3.90 & -2.075 & 70.4 & 82.0 & 77.2 \\
\hline & 6516.08 & 2.89 & -3.380 & 60.3 & 65.3 & 62.7 \\
\hline & 7224.46 & 3.89 & -3.243 & 18.8 & 25.5 & 21.5 \\
\hline & 7711.73 & 3.90 & -2.450 & 44.6 & 57.7 & 51.6 \\
\hline \multirow[t]{2}{*}{$\mathrm{Ni} \mathrm{I}$} & 5847.00 & 1.68 & -3.430 & 29.5 & 18.6 & 23.3 \\
\hline & 5857.75 & 4.17 & -0.390 & 67.2 & 67.5 & 73.8 \\
\hline
\end{tabular}


Table APPENDIX - Continued

\begin{tabular}{cccrrrr}
\hline \hline \multirow{2}{*}{ Ion } & $\lambda(\AA)$ & Ex. Pot. $(\mathrm{eV})$ & $\log g f$ & KW 23 & KW 208 & KW 335 \\
\hline & 6108.11 & 1.68 & -2.450 & 73.6 & 62.1 & 68.4 \\
& 6130.14 & 4.09 & -1.040 & 27.0 & 19.8 & 24.5 \\
& 6133.98 & 4.09 & -1.770 & 9.8 & 5.6 & 4.5 \\
& 6175.37 & 4.09 & -0.590 & 60.4 & 51.8 & 59.4 \\
& 6176.82 & 4.09 & -0.370 & 74.4 & 62.2 & 70.2 \\
& 6177.25 & 1.83 & -3.530 & 21.3 & $\ldots$ & 17.9 \\
& 6204.61 & 4.09 & -1.170 & 31.3 & 23.4 & 23.8 \\
& 6482.80 & 1.94 & -2.630 & 50.2 & $\ldots$ & $\ldots$ \\
& 6586.31 & 1.95 & -2.810 & 51.9 & 37.5 & 46.6 \\
& 6598.61 & 4.23 & -1.020 & 30.6 & 24.9 & 31.8 \\
& 6842.04 & 3.66 & -1.520 & 32.2 & 29.0 & 30.6 \\
& 7001.55 & 1.93 & -3.620 & 15.2 & 10.3 & 11.4 \\
& 7122.21 & 3.54 & -0.050 & 122.4 & 105.6 & 115.0 \\
& 7385.24 & 2.74 & -2.070 & 54.4 & 46.4 & 52.4 \\
& 7393.61 & 3.61 & -0.040 & 108.3 & 100.9 & 107.3 \\
& 7414.51 & 1.99 & -2.440 & 77.2 & 57.8 & 70.0 \\
& 7422.29 & 3.63 & -0.010 & 109.4 & 100.7 & 104.9 \\
& 7525.12 & 3.63 & -0.690 & 81.2 & 71.5 & 79.1 \\
& 7555.61 & 3.85 & +0.060 & 103.0 & 93.7 & 102.7 \\
& 7574.05 & 3.83 & -0.630 & 75.3 & 65.5 & 69.0 \\
& 7714.31 & 1.93 & -1.800 & $\ldots$ & 98.8 & 113.8 \\
& 7715.59 & 3.70 & -1.140 & 61.9 & 55.7 & $\ldots$ \\
& 7727.62 & 3.68 & -0.150 & 106.5 & 90.2 & 100.0 \\
& 7748.89 & 3.70 & -0.180 & 101.8 & 93.1 & 95.8 \\
\hline \hline & 6435.8 & -0.830 & 3.8 & $\ldots$ & $\ldots$ \\
& 6613.73 & 1.75 & -1.110 & 17.6 & 11.8 & 16.4 \\
& & 1.84 & -0.570 & 40.0 & 40.0 & 36.0 \\
\hline
\end{tabular}


